\title{
Towards a Singing Voice Multi-Sensor Analysis Tool: System Design, and Assessment Based on Vocal Breathiness
}

\author{
Evangelos Angelakis*(D), Natalia Kotsani (D) and Anastasia Georgaki \\ Laboratory of Music Acoustics and Technology (LabMAT), Music Studies Department, National and Kapodistrian \\ University of Athens, 15784 Athens, Greece; nkotsani@corelab.ntua.gr (N.K.); georgaki@music.uoa.gr (A.G.) \\ * Correspondence: angelakisv@music.uoa.gr
}

Citation: Angelakis, E.; Kotsani, N.; Georgaki, A. Towards a Singing Voice Multi-Sensor Analysis Tool: System Design, and Assessment Based on Vocal Breathiness. Sensors 2021, 21, 8006. https://doi.org/10.3390/ s21238006

Academic Editor: Antonio Guerrieri

Received: 1 September 2021

Accepted: 19 November 2021

Published: 30 November 2021

Publisher's Note: MDPI stays neutral with regard to jurisdictional claims in published maps and institutional affiliations.

Copyright: (c) 2021 by the authors. Licensee MDPI, Basel, Switzerland. This article is an open access article distributed under the terms and conditions of the Creative Commons Attribution (CC BY) license (https:/ / creativecommons.org/licenses/by/ $4.0 /)$.

\begin{abstract}
Singing voice is a human quality that requires the precise coordination of numerous kinetic functions and results in a perceptually variable auditory outcome. The use of multi-sensor systems can facilitate the study of correlations between the vocal mechanism kinetic functions and the voice output. This is directly relevant to vocal education, rehabilitation, and prevention of vocal health issues in educators; professionals; and students of singing, music, and acting. In this work, we present the initial design of a modular multi-sensor system for singing voice analysis, and describe its first assessment experiment on the 'vocal breathiness' qualitative characteristic. A system case study with two professional singers was conducted, utilizing signals from four sensors. Participants sung a protocol of vocal trials in various degrees of intended vocal breathiness. Their (i) vocal output, (ii) phonatory function, and (iii) respiratory behavior-per-condition were recorded through a condenser microphone (CM), an Electroglottograph (EGG), and thoracic and abdominal respiratory effort transducers (RET), respectively. Participants' individual respiratory management strategies were studied through qualitative analysis of RET data. Microphone audio samples breathiness degree was rated perceptually, and correlation analysis was performed between sample ratings and parameters extracted from CM and EGG data. Smoothed Cepstral Peak Prominence (CPPS) and vocal folds' Open Quotient (OQ), as computed with the Howard method (HOQ), demonstrated the higher correlation coefficients, when analyzed individually. DECOM method-computed OQ (DOQ) was also examined. Interestingly, the correlation coefficient of pitch difference between estimates from CM and EGG signals appeared to be (based on the Pearson correlation coefficient) statistically insignificant (a result that warrants investigation in larger populations). The study of multi-variate models revealed even higher correlation coefficients. Models studied were the Acoustic Breathiness Index (ABI) and the proposed multiple regression model CDH (CPPS, DOQ, and HOQ), which was attempted in order to combine analysis results from microphone and EGG signals. The model combination of $\mathrm{ABI}$ and the proposed $\mathrm{CDH}$ appeared to yield the highest correlation with perceptual breathiness ratings. Study results suggest potential for the use of a completed system version in vocal pedagogy and research, as the case study indicated system practicality, a number of pertinent correlations, and introduced topics with further research possibilities.
\end{abstract}

Keywords: biomedical signal acquisition; singing voice; data processing; breathiness; electroglottography; vocal mechanism; respiratory transducer; fundamental frequency estimation

\section{Introduction}

Singing is the result of certain neuromuscular functions pertaining to the vocal mechanism of the human body. Singing voice acoustic and perceptual properties have been studied since Ancient Greek times, mainly by Aristotle, Aristoxenus and Dionysius of Halicarnassus, and subsequently by Porphyrios, Nicomachus, and Cleonides [1]. This domain's study has been continuing, and a plethora of modern-day research works have been actualized regarding its anatomical, physiological, and acoustical properties [2], however there is still much to be uncovered [3]. 
One of the features that seems to require further investigation is singing voice overall quality assessment, which entails the quantification of individual perceptual voice attributes that define it, as "no agreed-upon method currently exists for objective measurement of perceived voice quality" [4] (p. 457). As a result, perceptual evaluation is still an important factor in both voice disorder assessment and measurement instruments result comparison, despite the extensive research realized and validity concerns [5]. The quantification and understanding of such qualitative measures is also important to vocal education, as well as to early diagnosis of voice misuse and prevention of relevant vocal health issues in professional singing voice users (singers, actors, music teachers, etc.).

In order to achieve a higher level of understanding of the singing voice, and to facilitate a more precise vocal pedagogy and rehabilitation, it would be useful to research and further uncover the correlations between the vocal mechanism parts' biomechanical functions and their resulting perceptual vocal properties.

\subsection{Singing Voice Acoustic Analysis}

A common acoustic model of the vocal mechanism involves the distinction of three parts: (a) the power source, or exciter (breathing system), (b) an oscillator (vocal folds), and (c) a resonator/filter (vocal tract) [6]. The resulting output is the auditorily perceived vocal timbre, which is defined by the contents of the vocal spectrum. This spectrum can be described, in the time domain, as the convolution of the glottal (vocal folds) pulse signal and the vocal tract filter impulse response [7,8]. Such an independent source-filter model of the vocal instrument (and the subsequent described convolution result) can be an "appropriate simplification" [7] (p. 2733) of a more complex interactions system.

The voice audio signal is easily acquired through a microphone, and its analysis can reveal quantifiable evidence on a number of the vocal perceptual characteristics. Fast Fourier Transform (FFT) as well as Logarithmic analysis methods on the spectrum of this microphone signal (Cepstral Peak Prominence (CPP), Smoothed Cepstral Peak Prominence (CPPS), Mel Frequency Cepstral Coefficient (MFCC), etc.), provide us with the potential to evaluate glottal events and vocal tract resonances separately [9] (for a brief introduction to CPP and CPPS cepstral measures please refer to Section 3.4).

\subsection{Multi-Sensor Singing Voice Assessment}

However, a more precise study of the voice could be achieved through combinatory analysis of the above vocal sound measures in conjunction with the respective vocal mechanism kinetic procedures. Such a venture requires the employment of more complex systems. In a previous positional/review journalpaper, analyzing the reported decline of operatic singing quality, as well as the challenges and recourse of vocal pedagogy [10], we have suggested the need for development of research and education tools, utilizing multiple sensors to transduce data regarding primary kinematic and acoustic functions of the vocal mechanism. We also proposed that a series of experiments be actualized, aiming towards the clarification of biomechanical-acoustical correlations.

The aim of the present work Authors: Changed for text clarity is multifold: (a) the initial configuration of a modular, multi-sensor singing voice analysis system; (b) the application of that system's pilot study to research aspects of the breathiness characteristic in singing; and (c) the utilization of the aforementioned research's data for the evaluation of vocal breathiness possible effects on the accuracy of an under-development vocal tuner software.

The sensors incorporated to the above system were an Electroglottograph (EGG), two Respiratory Effort Transducers (RET), and a Condenser Microphone (CM), while its pilot study investigated possible system issues regarding: system and sensor usability, singeruser convenience/task-hinderance level, compatibility, connectivity, signal acquisition, data recording synchronization, and signal noise levels. The vocal breathiness characteristic in singing was examined through a multi-faceted scope. Evaluation measures used were Acoustic Breathiness Index (ABI), CPPS, vocal folds Open Quotient (OQ) — computed both 
with the Howard (HOQ) and the DECOM methods (DOQ) - , singing voice Fundamental Frequency $\left(f_{o}\right)$, phonation duration, "respiratory management strategies" (i.e., participants' individual respiratory management strategies in singing, through - either conscious or unconscious- muscular control of the breathing system), and breathiness level perceptual evaluation. A new multivariate index, $\mathrm{CDH}$ (CPPS, DOQ, HOQ), was also proposed.

As mentioned above, experimental data extracted during the above pilot study were also used to evaluate the efficiency of a microphone as sensor for pitch tracking purposes in the case of users with varying degrees of vocal breathiness. This was realized by assessing the deviation of Praat's [11] pitch-tracking algorithm results between (1) CM signal and (2) the respective EGG signal, in 'distinct levels of intentionally breathy singing voice'. This algorithm has been used in the $f_{\text {habitual }}$ frequency tool [12] and is intended for use in Vocal Tuner Tool, both being parts of the 'Assistance for students in Singing and Music Aesthetics' (ASMA) project, involving the authors of the present work.This new tool, estimating voice fundamental frequency in combination with various spectral features of the voice, is designed for use from school children and their teachers, without requiring the added presence of medical or vocal experts (perceptually detecting possible vocal breathiness prevalence of users). Results from a performance study on seven established pitch detection algorithms [13] have shown indications of a "pitch error" increase in pathological voices. Breathiness has been reported to have a $24.2 \%$ percent prevalence in a study of 71 children $3-9$ years old [14], and $37.71 \%$ prevalence in a study with 70 children aged 6-10 [15]. It was therefore considered important to test the algorithm in breathy singing, and to compare its deviation between application on CM and EGG signals. It was hypothesized that an EGG signal could be able to provide more accurate fundamental frequency estimation data in cases of breathiness prevalence, as (a) breathy voice air turbulence noise is not a part of the EGG signal, (b) vocal tract 'filtering' on voice signal is not actively affecting the glottal pulse frequency, and (c) EGG signal is impervious to ambient noise, which could mask the acoustic voice signal during field experimentation with breathy voices (where pitch strength is already low [16]).

\subsection{Related Work}

Multi-sensor singing voice analysis is a field that is currently expanding. Examples of software for combined EGG and microphone signal analysis are 'PhaseComp' [17] and 'VoceVista Video Pro' [18], which have been developed by EGG device manufacturing companies. Additionally, the open-source voice analysis software Praat [11] has incorporated scripts for working with an EGG signal, while the twocompanion software 'VoiceSauce' [19] and 'EGGWorks' [20] can be fused to export combined analysis results for audio and EGG signals. Research on the singing voice realized using multiple sensor input is not very common. Watson and Hixon [21] studied opera singers respiratory kinematics using microphones, magnetometers, and video recording, while Salomoni et al. [22] used a microphone, respiratory inductance plythesmography bands, and a pneumotachograph. A 2018 study [23] compared measurements between an electret condenser microphone, a sound level meter, and a piezoelectric contact microphone, assessing (among others) pitch inaccuracies and cepstral measures of singing voice quality estimation in 14 professional singers. Furthermore, a section of the international UNESCO 'iTreasures' project for the preservation of 'Intangible Cultural Heritage' [24] resulted in the creation of a platform for the capturing and analysis of rare singing techniques (i.e., Corsican Paghjella, human beat box, and Byzantine chanting). This platform employed data capturing from a microphone, an EGG, an imaging camera, a breathing belt, an ultrasound module, and piezoelectric accelerometers [25]. Another study on singers employing both invasive and non-invasive sensors regarded the definition of "supported singing voice" through the comparison of the singers' self-perception of supported and un-supported singing with quantitative measures [26]. Additional sensors that can be (or have already been) used for singing voice biofeedback include spirometers, skeleton tracking cameras, videokymograph, and functional magnetic resonance imaging devices [10]. 
Although the studies listed here performed a multi-sensor analysis, a significant number of researchers have studied the singing voice with the use of solely accelerometers, which can be employed for the examination of various voice-related measures, such as fundamental frequency, open quotient, glottal airflow [27], and subglottal pressure [28], that are traditionally captured with a microphone, an EGG, or airflow measurement devices. Accelerometers have also been used in ambulatory voice monitors for analysis of singers' vocal qualities, vocal habits, phonotrauma risk assessment $[29,30]$, and detection and classification of singing [31].

A recent study [32] on 20 professional singers used a videonasoendoscopic camera system, a laryngostroboscopic system, an EGG, and a microphone to "assess and quantify singers' strategies for adding air to phonation to sound 'breathy' in a healthy manner" [32] (p. 1). Finally, the study utilizing the set of sensors closest to the one described in the current work was published by Ternström et al. [33], and reported findings from eight trained female singer regarding the effects of relative lung volume on the EGG waveform.

Vocal 'breathiness' is a qualitative vocal characteristic that has been studied quite extensively during the last decades [32,34,35]. It was chosen as an assessment case study due to (a) its association with all three parts of the vocal mechanism, (b) its connection to overall voice quality [36], and (c) its prevalence in children with dysphonia, a factor which the authors wish to control for in ASMA project. The Consensus Auditory Perceptual Evaluation of Voice (CAPE-V) standardized clinical protocol definition of the breathiness vocal attribute is "audible air escape in the voice" [37] (p. 127), while the ABI introductory study [35] specifies the characterization of breathy voice by a "turbulent noise during phonation with excessively high frequency resulting from air leakage during glottal closure" (p. 511.e11). This attribute is of interest to vocal pedagogy [32] and linguistics [38], as it is a quality-defining perceptual characteristic [34], but also important to phoniatrics, as it has also been connected to pathological conditions, such as vocal nodules, vocal fold bowing, paralysis or paresis of the recurrent laryngeal nerve, and acute laryngitis [35]. Breathiness has long been reported as "quantitatively related" to insufficient vocal fold closure [39] (p. 5), resulting in continuous air leakage through the glottis (i.e., the opening/space between the two vocal folds). Abduction (opening) and adduction (closing) of the vocal folds is a determining factor for phonation and text articulation [6]) during phonation. This makes breathiness a particularly important characteristic, as insufficient vocal fold closure may imply a medical condition [40] but could also result in one [41]. Despite the apparent multidisciplinary interest in the 'breathy' voice quality, there are still publications reporting a lack of adequate data correlating the 'breathy voice' perceptual, qualitative feature, to the pertinent biomechanical functions and the resulting acoustical, quantitative measures [32].

A 'breathy' voice may be tolerable, or even desired as a timbral option in some music genres, such as Jazz, or some 20th-21st century music compositions using 'Extended Vocal Techniques' (a term that is used to include phonation modes or experimental techniques that deviate from the norm for Western classical singing, such as multiphonics, overtone singing, ingressive singing, growling, etc.) [42] (pp. 21-22). This intentional use of the breathiness characteristic is sometimes referred to as "air added to the voice", to distinguish it from involuntary breathy voice production [32] (p. 1). This study makes use of the term breathy as the perceptual characteristic, without differentiating between these conditions. Unintentional vocal breathiness is usually considered as a negative characteristic in many music styles, such as operatic singing [43]. This is due to breathiness being connected to relatively low vocal intensity (opera singers have to be clearly audible in large auditoriums over the sound of a symphonic orchestra without amplification) and low "pitch strength" [16], the latter implying the presence of a wide-band noise [44] from the leaking air. Prevalence of this noise indicates the breathiness perceptual degree and has been studied using various acoustic measures [35].

The following is a brief overview of the related work pertaining to vocal breathiness analysis. Pongweni corrected typo in name [45] studied the effect of breathy voice on Shona vowels using the amplitude, pitch and formants. Fritzell et al. [39] concluded that a 
quantitative relationship exists between breathy voice quality and glottal insufficiency in pathological voices according to perceptual judgments. Scherer and Titze [34] extracted the abduction quotient $\left(Q_{a}\right)$ from electroglottographic recordings in speech and singing tasks in different voice qualities (breathy, normal, and pressed or constricted), concluding that the abduction quotient decreases from breathy to pressed voice and suggesting that it corresponds to the effective glottal width. Hartl et al. [46] showed that unilateral vocal fold paralysis (UVFP) related breathiness was best correlated with airflow measurements and that 10 of the 14 objective acoustic and aerodynamic parameters successfully distinguished patients with UVFP from the control group. Shrivastav and Sapienza [47] compared several measures obtained from the output of a previous auditory model to the perceptual ratings of breathiness (for the Kay Elemetrics Disordered Voice database) using multidimensional scaling techniques, concluding that the perceptual ratings of breathiness were best predicted by the partial loudness of the periodic signal and stating the importance of an auditory model as a signal processing front-end in obtaining objective measures of voice that correspond closely with listeners' perception of breathiness. Teixeira et al. [48] presented a non-invasive procedure for automatic diagnosis of pathologies of the larynx using jitter, shimmer (correlating it with breathiness), and HNR parameters, and proposed threshold values for pathological voices. Fraile and Godino-Llorente [49] highlighted the reliability of CPP in dysphonia detection and its extended use to the evaluation of overall voice quality, commenting, however, that this particular measure is not sufficient for predicting voice quality aspects related to breathiness.

Borsky and Guðnason [50] classified five different voice types (modal, breathy, rough, soft, and pressed) with Gaussian Mixture Model (GMM) from speech samples using an electroglottograph and extracting the MFCCs. Barsties v. Latoszek et al. [35] proposed a nine-variable acoustic model for the multiparametric measurement of breathiness, the Acoustic Breathiness Index (ABI), in dysphonic voices using stepwise multiple regression analysis. Kadiri and Yegnanarayana [51] classified breathy, modal, and tense voice using support vector machines (SVMs) and the zero-time windowing method, optimizing the detection of phonation type when combining various acoustic and glottal features. Aaen et al. [32] assessed air added to the voice (i.e., a singer's strategy of adding air to create a vocal breathiness effect in a healthy voice) by extracting various features from laryngostroboscopic imaging and electroglottograph data, and observed a glottal gap along the edge of the length of the vocal folds, as well as significant differences in various acoustic parameters. Tylečková and Skarnitzl [52] proposed quantitative ranges of voice quality parameters on non-pathological voices based on speech reading tasks in Czechs, concluding that the estimated CPP values describe modal (e.g., non-breathy) phonation. Barsties v. Latoszek [40] quantifyied evidence for the diagnostic accuracy of ABI, in terms of sensitivity and specificity, from 34 research works, confirming the ABI's robustness and validity. Murton et al. [53] used CPP to evaluate clinical voice concluding in CPP cutoff thresholds indicating the presence of voice disorders.

A recent publication indicated that breathiness studies have been (a) mostly concerned with speech, (b) included singers as participants in a percentage seemingly lower than $2 \%$ (35 out of 1923 in the 29 reviewed studies), and (c) rarely involving female and male singers concurrently [32].

The system-in-development described and tested in the present publication will be, when completed and to the best of our knowledge, one of the few non-invasive tool attempts for the voice to combine acoustic, glottal, and respiratory signal acquisition from dedicated sensors. The assessment experiment and analysis included measures that are rarely studied against each other. A strong correlation has been hypothesized between perceptual breathiness and CPPS, as well as between breathiness and OQ. The Acoustic Breathiness Index was computed and a new multivariate regression model (CDH) was introduced, to associate data results from CM and EGG signals for breathiness prediction, while the possibility of combinatory analysis, using $\mathrm{ABI}$ and $\mathrm{CDH}$, is also explored. 
Reports of transglottal airflow increase up to $~ 60 \%$ in breathy vowel phonation [54] also lead to the assumption of a decrease in maximum phonation duration with breathiness perceptual degree.

Furthermore, following the indications for pitch detection algorithm accuracy comparison between healthy and pathological voices [13], a secondary hypothesis was made. This predicted a non-significant deviation when comparing results from the Praat pitch detection algorithm between application to EGG and microphone signals of non-breathy singing, but a significant deviation in respective results for breathy singing.

Correlation analysis appeared to confirm the aforementioned hypotheses, disproving only the hypothesized accuracy decrease in microphone signal fundamental frequency estimation in breathy singing, when compared to an EGG signal.

The innovative aspects of this work consisted mainly of (i) the design and testing of a developing multi-sensor architecture to capture various parameters of the singing voice; (ii) the comparative study of a specific pitch detection algorithm effectiveness in CM and EGG signals in increasing singing voice breathiness conditions; and (iii) the introduction of $\mathrm{CDH}$, which is a new multi-variate regression model for the prediction of breathiness in the singing voice, combining data from both microphone and EGG signals, which revealed significant results for our dataset.

\section{Materials and Methods}

The system setup described here is being designed as a portable, non-invasive, modular monitoring system for signal acquisition, synchronization, and offline processing of multiple sensors, transferring data from all three main parts of the vocal mechanism (breathing system, phonatory system, and vocal tract). A distinct type of sensor was used for each of the above mechanism parts. The exception to this was the monitoring of the vocal tract with the use of a microphone, which can record the final vocal output, resulting from vocal tract filtering on the vocal folds pulse signal. However, as mentioned above, microphone signal data analysis can reveal useful information distinctive of the vocal tract modifications and filtering. All sensors used in this initial system version could sent data for recording and processing independently, through their respective signal acquisition units, without direct sensor inter-communication.

As portability was a primary prerequisite for the equipment choice, the entire experiment equipment (excluding the microphone stand) was fitted and held in a large photography backpack, as shown in Figure 1. This will allow for future field experiment measurements to be conducted.

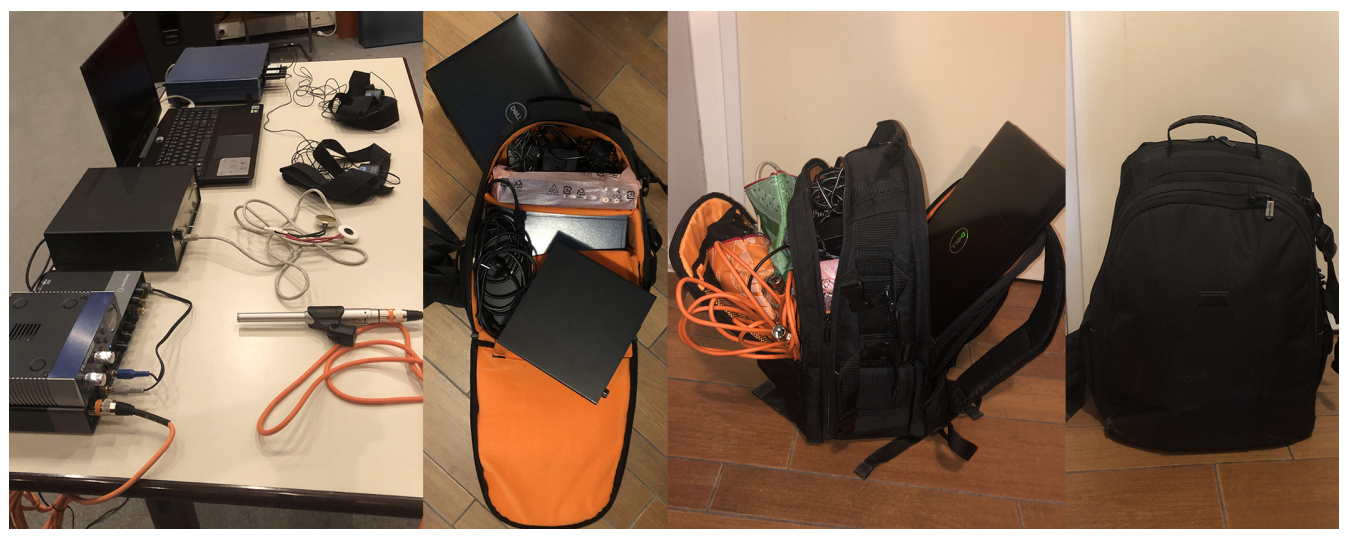

Figure 1. The entire experiment setup equipment, both ready-for-use (left) and fitting a backpack (center and right).

\subsection{Breathing System Sensors}

The breathing system was monitored by two respiratory effort transducers (Biopac SS5LB) connected to a Biopac MP35 Signal Acquisition Unit (Biopac Systems, Goleta, CA, USA). Each of the transducers was connected to a soft, adjustable nylon strap and 
measured the circumference expansion and contraction of a certain torso region. They were selected for the experiment as they claim to "measure extremely slow respiration patterns with no loss in signal amplitude while maintaining excellent linearity and minimal hysteresis" [55]. Additionally, SS5LB belts are lightweight, extremely unobtrusive for the singers, and sterilizable. One strap was placed around the thoracic region (RETt), about $5 \mathrm{~cm}$ underneath the armpits (as suggested by the manufacturer), and a second was placed around the abdominal region (RETa), right under the navel. Following the SS5BL Instruction Manual, the straps were adjusted to have a slight tension at maximum expiration state for each participant. RET placement also followed the respective model used by Watson and Hixon [21].

\subsection{Phonation}

The vocal folds' behavior was monitored with the use of an Electroglottograph (EGG). The EGG is a non-invasive sensor that has been used extensively during the last six decades for studying various aspects of laryngeal voice production, and its application has resulted in a number of contributions to the science of the singing voice [56]. It employs the use of two electrodes, which are placed bilaterally on the larynx (usually with the help of an external soft strap). The EGG provides output regarding the relative contact area of the vocal folds [57] in real-time, by measuring electric conductivity of a small current, with a frequency commonly ranging from $300 \mathrm{kHz}$ to several megahertz [58]. For the present experiment, the EG2-PC electroglottograph by Glottal Enterprises was used. This model additionally employs a 'Laryngeal tracking indicator', which can be utilized to ensure correct electrode placement and minimize vertical larynx movements' effect on performance [57]. The unit is powered with the help of two internal rechargeable batteries and remains unplugged during experimentation to allow for maximum line noise reduction and transportability. To achieve optimal signal acquisition and minimize noise, the EGG was used, making sure to maintain a small interelectrode angle and distance (as shown in Figure 2), following the guidelines by I. Titze [59] and the instructions for the EGG model used in our experiment by A. Michaud, including methods by N. Henrich and B. Gautheron [60]. The latter instructions were also followed for experimental conditions, device settings (with the exception of setting the EGG output level to 'High'), device use, and electrode alignment, whereas electrodes were held in place only by hand to allow for maximum vocal freedom.

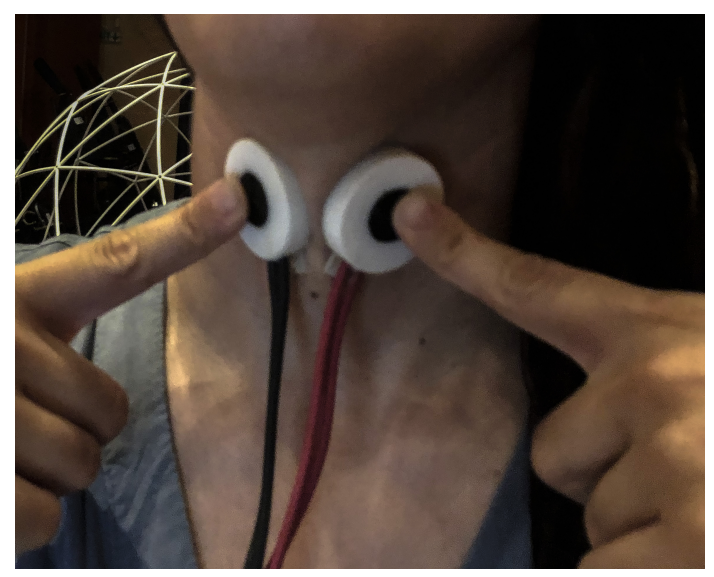

Figure 2. Participant displaying EGG electrode placement and alignment.

\subsection{Vocal Output}

The Behringer ECM8000 measurement microphone was use to capture the vocal output. It is an omnidirectional microphone with flat frequency response at a $15 \mathrm{~Hz}$ to $20 \mathrm{kHz}$ range and requires phantom power $(+15$ to $+48 \mathrm{~V})$. The microphone was placed at a $40 \mathrm{~cm}$ distance from the singer's mouth and its signal was passed through a PreSonus TubePre professional microphone tube preamplifier, using XLR cables. 


\subsection{Connections}

Both the EGG signal and the pre-amplified microphone signal were input to two distinct channels of a Steinberg UR44 external sound card. The sound card was connected to a dedicated Dell G5 15 Laptop via a USB Type-C cable.

\subsection{Software and Sensor Signal Recording}

The (a) microphone audio and (b) EGG input signals were recorded at $48 \mathrm{kHz} / 24$ bit PCM (Pulse-Code Modulation audio format), following the suggestion of a previous study [32] for a minimum of $16 \mathrm{kHz}$ recordings, and using the Audacity Open Source multi-track audio editor and recorder for Windows (Version 3.03). The resulting audio was exported in files with .wav extension. The two Biopac SS5LB sensors signal, passed through a Biopac MP35 Acquisition Unit, which output the acquired signals to the same laptop via USB connection, as depicted in Figure 3. This was then recorded with the use of the Biopac Student Lab (BSL) 4.1 Pro Software, using the software default sensor settings (not the SS5BL settings). Waveforms from SS5LB sensors signal were also exported in .wav files to allow for maximum compatibility in analysis with the microphone and EGG signal files. In order to ensure the optimum synchronization of the four sensor signals, being recorded by two distinct software programs, a script was written in the open-source scripting language AutoHotkey (version 1.1.33.09). This enabled recording to commence in both programs simultaneously (using a single keyboard key), and to thus result in exported files with common time-stamps that could be synchronized more accurately for the purpose of data analysis.

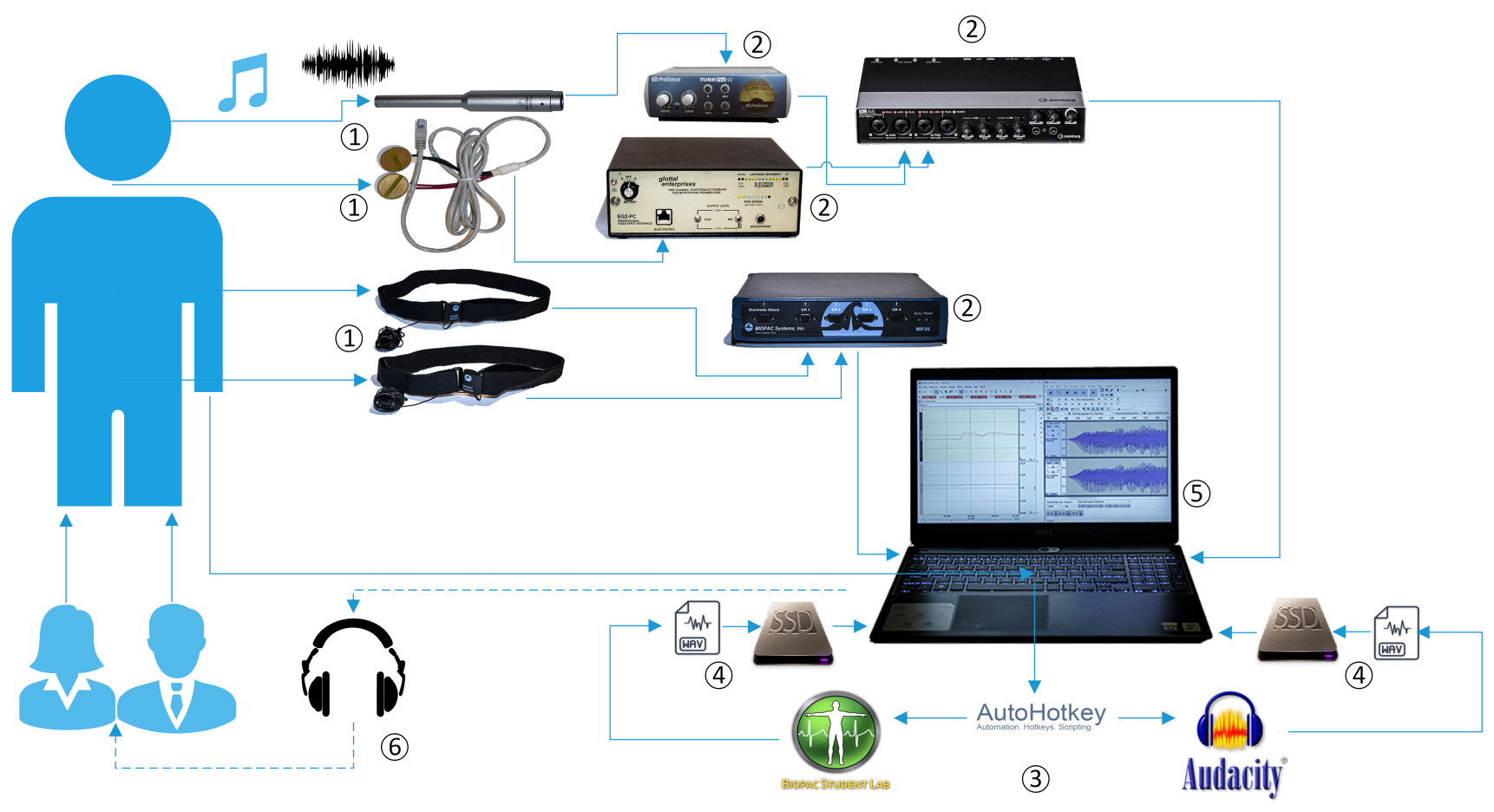

Figure 3. System Architecture: (1) Sensors. (2) Signal Acquisition and Amplification Units. (3) Data Recording Synchronization. (4) File Export and Storage. (5) Data Analysis. (6) Post hoc Perceptual Evaluation.

\subsection{Ambient and Hardware Noise}

All experiment measurements took place in a soundproof recording room at the recording studio of the Laboratory of Music Acoustics and Technology (LabMAT) of the Music Studies Department of the University of Athens. In order to obviate a substantial portion of possible interference and noise during measurement recordings, a laptop computer 
was setup to record using internal battery power and optimized for minimum-to-zero fan noise. Furthermore, the external sound card was powered from the laptop using a USB-C connection, while the EGG processor was powered by two internal batteries. The EGG manufacturer claims that their "electroglottographs produce very low-noise EGG waveforms" (https:/ / www.glottal.com/Electroglottographs.html (accessed on 27 November 2021)). All three devices were disconnected from external power sources and current transformers.

The Signal-to-Noise Ratio (SNR) was $45 \mathrm{~dB}$ for the recorded CM signals and $30 \mathrm{~dB}$ for the EGG signals. This should allow the extraction of valid EGG results (SNR $\geq 30 \mathrm{~dB}$ ) and $\mathrm{CM}$ results with a theoretical measurement accuracy of $99 \%$ (SNR $\geq 42 \mathrm{~dB}$ ), according to reference studies [61,62]. For the purposes of this particular study, the RET signal was analyzed qualitatively through signal waveform, where no noise seemed to visually interfere. Due to the nature of respiratory muscles movement and relatively very low speeds, RET signal frequencies of interest reside in the range of under $10 \mathrm{~Hz}$ (normal respiratory cycles have a frequency of approximately $0.2-0.3 \mathrm{~Hz}$, while frequencies higher than $1 \mathrm{~Hz}$ "are commonly registered during high-intensity exercise" [63]). For system test purposes, a software low-pass filter [64] was applied in Audacity, using a relatively high threshold of $40 \mathrm{~Hz}$, in order to overcompensate for any excessively fast muscle movement, but cutting off possible interference of local electrical current $(50 \mathrm{~Hz})$. This filtering resulted in elimination of any noise from mechanical or electrical sources upwards of $40 \mathrm{~Hz}$, as shown in Figure 4.

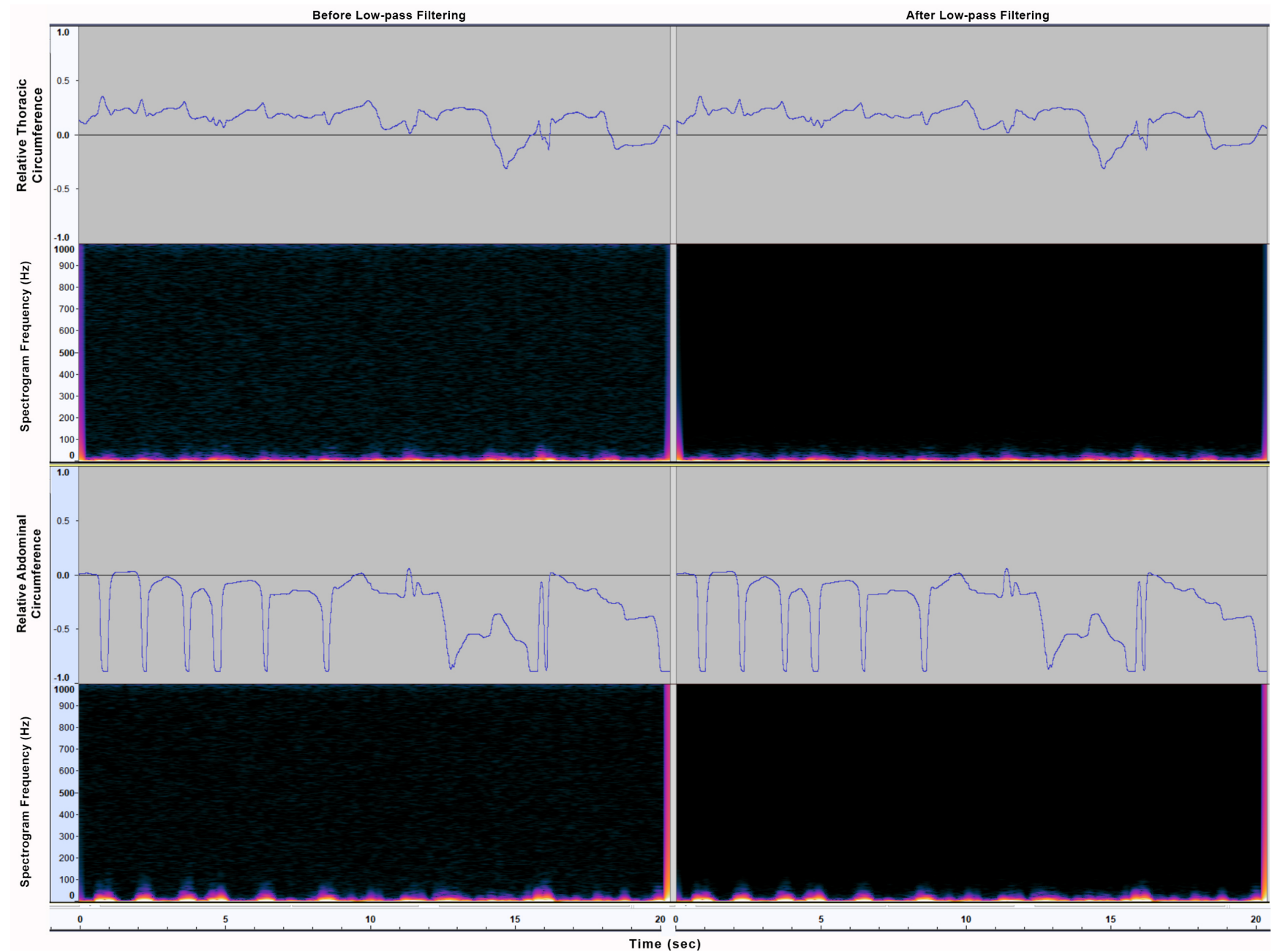

Figure 4. Waveforms and spectrograms of the two (top and bottom) RET sensors' signal low-pass filter application for noise removal, in a sample of intentionally excessive muscle movement (Left: before filtering; Right: after filtering). 


\subsection{Experiment Participants}

Professional operatic singers have been used as participants in research studies pertinent to the singing voice, as their extremely demanding profession [10] requires extensive training and practice. The genre generally known as "operatic singing" or "Western classical singing" is furthermore one of the most demanding types of vocal music. It encompasses a huge variety of sub-genres of many Western vocal traditions, ranging from the 17th century to today. It requires singers to employ a high skill level pitch precision, vocal range, volume/audibility, lyrics discernibility, voice health and stamina, vocal agility/flexibility, musicality, stylistic proficiency, acting capability, etc. It is a trait often compared to elite athleticism. This has been reported to promote a higher degree of kinesthetic control [65] and enable the voluntary isolation or prevalence of distinct feedback modalities (auditory, kinesthetic/somatosensory) $[65,66]$. Two participants took part in this study (1 female, 1 male-authors NK and EA, respectively). Both were professional singers with classical training and professional experience (21 and 28 years, respectively) both as singers and teachers of singing. Participants stated that they perceived their vocal instrument to be in good health and that, in the past, they have both had stroboscopic medical evaluations that had revealed no chronic vocal health issues. The data are available in a publicly accessible repository (https://github.com/nataliakotsani/Singing-Voice-Multi-Sensor-AnalysisTool/tree/main/DATASET (accessed on 28 November 2021)).

\subsection{Experimental Protocol}

The participants used the recording room one at a time. Participants were allowed to commence at their own convenience and follow the protocol at their preferred pace, pausing between trials, as they needed. The protocol of trials was discussed and was available for them in a printed page. The protocol consisted of vocal trials on distinct voluntary controlled degrees of vocal breathiness in singing. Three breathiness cases were studied: (1) Non-Breathy singing Voice (NBSV), (2) Breathy singing Voice (BSV), and (3) 'Gradual' Breathiness singing Voice (GBSV), where the breathiness characteristic had to slowly and gradually be changed from non-breathy to breathy, quantifying data for the varying degrees of this perceptual voice feature. Each of the above cases were recorded on three degrees of vocal intensity (medium, high, low $-\mathrm{m}, \mathrm{h}, \mathrm{l}$, respectively), resulting in a total of nine experimental conditions. For each of these nine conditions, the participants were asked to sing the following six trials, sustaining their sound for as long as it was comfortable for them, aiming towards their maximum duration at optimum vocal efficiency. Trials were (a) single sustained note on a comfortable tone, (b) single sustained note on a high tone, (c) single sustained note on a low tone, (d) ascending vocal glissando (2-octaves minimum), (e) descending vocal glissando (2-octaves minimum), and (f) ascending and descending 2-octaves arpeggio. This resulted in a total amount of 54 distinct trials for each participant. Each participant's complete trial run was recorded as one incessant file, including failed and repeated trials. Participants were instructed to cough lightly employing a simultaneous engagement of the abdominal region muscles before commencing the trial run, as well as right after concluding the last trial. This served the purpose of post hoc data synchronization via actively generated common events, which is reported in the literature as a common approach [67]. Participants stated orally, for the recording, the exact condition for each trial before commencing it.

\section{Analysis}

The section that follows describes in detail the way in which the experiment data was handled, rated, classified, and subsequently analyzed (a) quantitatively, (b) qualitatively, and (c) statistically.

\subsection{Data Handling and Analysis Parameters}

Total experiment time for participants 1 and 2 (P1 and P2) was 23 min $14 \mathrm{~s}$ and 16 min $42 \mathrm{~s}$, respectively. Experiment data were exported into $48 \mathrm{kHz} / 24$ bit .wav files 
and processed in Audacity. Audacity inherently performed lossless files conversion from 24-bit integer to 32-bit floating-point. Initial file processing involved separation of 32-bit stereo audio files into two separate 16-bit monophonic files (one for each sensor) and the subtraction of aborted trials, silent intervals, and oral trial descriptions, resulting in concatenated .wav audio files containing solely the experiment trials sound. Processed files had respective duration of $7 \mathrm{~min} 31 \mathrm{~s}(\mathrm{P} 1)$ and $7 \mathrm{~min} 44 \mathrm{~s}(\mathrm{P} 2)$ and were subsequently segmented each into multiple $2 \mathrm{~s}$ consecutive numbered .wav sample files, preserving the initial 16 bit-rate. Sample files were numbered from 000 to 224 for P1 and from 225 to 455 for P2. Microphone and EGG sound channels were processed simultaneously, creating an equal number of audio files for the EGG signal.

For the analysis, the dataset samples were considered as independent data points (as in corresponding studies of the literature [68]), instead of the subjects. The descriptive outcomes and the correlation factors, between the selected parameters and the perceptual breathiness ratings, were extracted using specific statistical libraries in python programming language, and the implementation is available in a public repository (https: / / github.com/nataliakotsani/Singing-Voice-Multi-Sensor-Analysis-Tool, (accessed on 28 November 2021); https:/ / doi.org/10.5281/zenodo.5732933 (accessed on 28 November 2021)) [69].

The selected features for the analysis were the Pitch Difference between estimations from the CM and the EGG signals, in both $\mathrm{Hz}$ (PD) and Mel Scale (MPD), the Smoothed Cepstral Peak Prominence (CPPS), the Open Quotient extracted with both the derivative of the EGG signal-DECOM-(DOQ) [70] and Howard (HOQ) [71] methods, as well as the Acoustic Breathiness Index (ABI) [35].

The correlations between the perceptual breathiness ratings and the above parameters were analyzed. Of these acoustic and glottal parameters, the ones that displayed higher correlations to breathiness ratings were CPPS, DOQ and HOQ. These were selected in an attempt to model the relationship between them and perceptual breathiness. Using multiple linear regression, a new index $(\mathrm{CDH})$ for the prediction of the breathiness rating was computed using the microphone and EGG signal results, through exclusively the CPPS, $\mathrm{DOQ}$, and HOQ parameters. In addition, the $\mathrm{CDH}$ index was experimentally combined with the ABI index, a combination that yielded the highest correlation coefficients for our dataset.

\subsection{Perceptual Evaluation}

The above 456 two-second microphone audio sample files were evaluated perceptually, using the same two experiment participants as judges, utilizing their expertise and experience as singing teachers [72]. The evaluation was performed using a 9 point discrete rating scale from ' 0 ' (absolutely no perceivable breathiness in the voice) to ' 4 ' (excessively breathy vocal sound with a barely discernible pitch), including half point values $(0.0,0.5$, $1.0, \ldots, 3.5,4.0)$. Both judges reported to have no permanent or impermanent hearing or vocal impediment. They evaluated all 456 samples (from P1 and P2), but each in a distinct randomized order, using separate online forms, to avoid influence for consecutive samples and priming effects, as well as to control for participant fatigue effect distribution.

Each participant evaluated the samples in four approximately 1-h sessions, with interposed intervals to further control for fatigue, using the same model of closed type headphones, and each at their own, personal environment.

The double attribute of the participants/raters was decided in order to ensure ability of the raters to perceptually discern between variable conditions of vocal breathiness. A previous intra-rater reliability and validity study on non-expert dysphonic patients [73] reported findings that self-rating of perceptual voice qualitative features (including breathiness) appeared to be consistent and partially valid. Partial validity in that study [73] was hypothesized to be attributed mainly to self-raters' lack of expertise, poor auditory discrimination and poor auditory memory skills prevalence in vocal patients, as well 
as psychogenic factors. These influences were considered to be non-existent in current research with healthy, expert participants.

In the work presented here, self-rating was predicted not to be significantly affected by biases as (a) audio samples were merely $2 \mathrm{~s}$ long, leading towards judgment for the specified vocal property, rather than overall singing quality; (b) during the recording experiment, participants were asked to sing trials utilizing various degrees of breathy voice (thus minimizing the response bias for a 'good' rating from themselves as judges); (c) samples were presented for judgment in separate computer-generated randomized orders, to disassociate them of any sense or knowledge regarding the intended target breathiness degree for each sample; (d) trial audio segmentation in 2-s samples, the large total of samples number (456), and a 15-day interval between measurements and perceptual judgment, was employed to help eliminate any associative memory between experiment trial expectancy and ratings; and (e) the judges had knowledge that participants' initial breathiness target degree was irrelevant to the study (as breathiness is a perceptual characteristic), that correlations between intended voice breathiness degree and perceptual rating was not being examined, and that statistical analysis would be based solely on the auditory rating and not initial target intention.

Evaluations comparison between judges for the total 456 samples, in the 9 point scale, revealed absolute judgment agreement in $63.8 \%$ of the samples $(n=291), 1$ point judgment deviation in $22.8 \%(n=104), 2$ to 3 point deviation in $11.9 \%, 4$ to 5 point deviation in $1.5 \%$ $(n=7)$, and $0 \%$ for 6 to 8 points deviation.

For the descriptive statistics outcomes, the above samples were classified according to their average breathiness perceptual ratings. Calculating the average from the two judges for each sample resulted in scores with a total of 17 values and a 0.25 step $(0.00$, $0.25,0.50,0.75, \ldots, 3.75,4.00)$. For comparability with previous studies that used a 5 -point evaluation [32,47], samples were then classified using a taxonomy of the above 17 values into five classes $(0,1,2,3,4)$ of three to four values per class, minding the first and last classes ( 0 and 4 , respectively) to include exactly three values each. The latter was opted in order to achieve a more uniform distribution of the sample population (see Figure 5), as the experimental breathiness target conditions were (a) NBSV (which should yield samples belonging mainly to class 0 ), (b) BSV (which should yield samples belonging mainly to class 4), and (c) GBSV (which should yield samples belonging to all classes, including 0 and 4). Consequently, the grouping used for the five respective classes was $<0.75, \leq 1.5$, $\leq 2.25, \leq 3.25, \leq 4$.

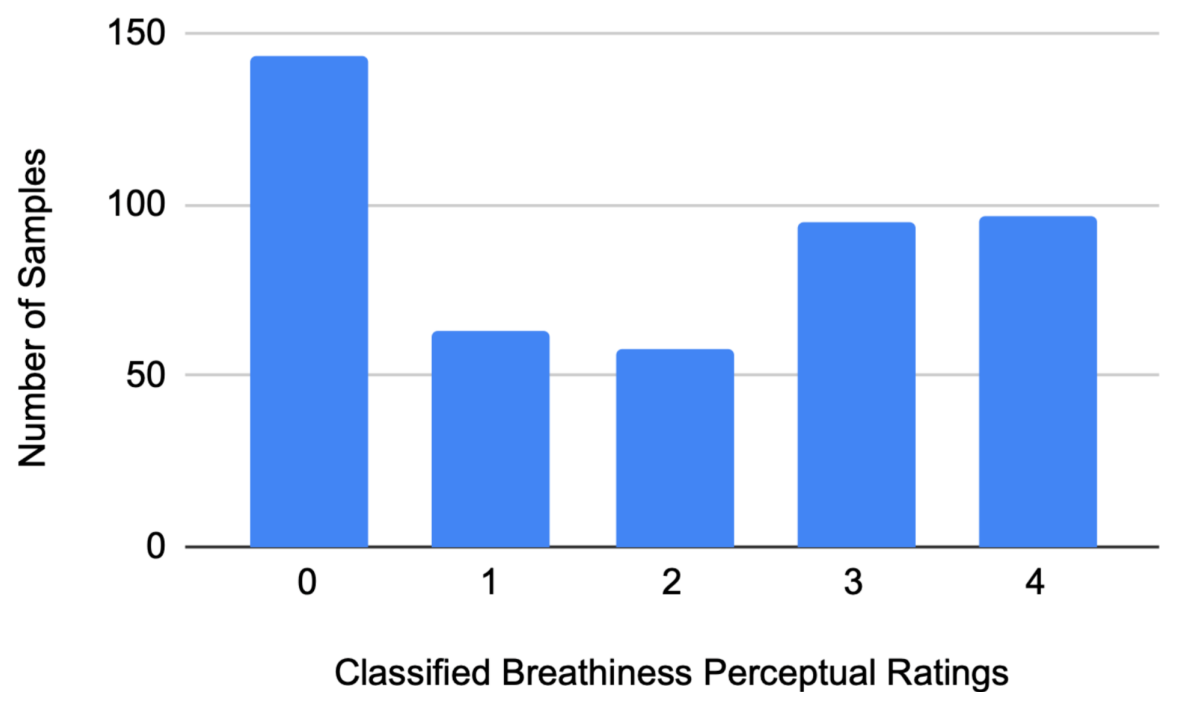

Figure 5. The numbers of samples per class. 


\subsection{Pitch Detection in Microphone and EGG Signals}

The aim of this experiment included the assessment of the Praat pitch detection algorithm, used also in the ASMA project for the $f_{\text {habitual }}$ frequency tool [12]. In previous works regarding the comparison between the microphone and the EGG signals, Vieira et al. [74] compared the acoustic and EGG signal jitter for dysphonic speakers, and Jang et al. [13] studied the comparative results of seven pitch detection algorithms for verification of adequacy in pathological voices.

For the comparison of the pitch detection algorithm between microphone and EGG signals we used our dataset and the python's parselmouth library [75] which provides an interface to the internal Praat [76] code, directly accessing Praat's C/C++ code. The Praat's pitch detection algorithm was used in the microphone and EGG signals, comparing the differences of the results as the breathiness evaluation values increased. A correlation analysis was conducted between the breathiness perceptual ratings and (i) the difference of the pitch detection algorithm results when applied over the CM and the EGG signals, and (ii) the absolute values of that same difference.

\subsection{CPPS of the CM Signal}

For the extraction of the CPP, a Fourier transform is applied to the logarithm power spectrum of a recorded sound wave. The relative amplitude of the cepstral peak in relation to the expected amplitude of the cepstral peak is then estimated, using linear regression. Intuitively, it has been mentioned that the CPP represents the degree of periodicity in the voice signal and higher CPP values emerging from well-defined harmonic structure [77], but, despite its extended use for overall voice quality evaluation, a definite explanation of "what CPP actually measures" [49] is still lacking.

Formally, given a signal $s(t)$, its real cepstrum, or power cepstrum, is equal to the Fourier transform of the logarithm of its power spectrum, according to the first definition of cepstrum [49]

$$
C_{r}(q)=\mathcal{F}\left\{\log |S(f)|^{2}\right\}
$$

where $S^{2}(f)$ is the power spectrum of the signal

$$
s^{2}(f)=\mathcal{F}\{E\{s(t) \times s(t-\tau)\}\}
$$

A variant of CPP, called smoothed CPP (CPPS), was selected for acoustic analysis in the present work. This variant utilizes smoothing operations added both in temporal and cepstral domains [38], and has been reported to provide higher correlation with breathiness [49]. More specifically, the modification of the CPP algorithm consisting of an additional processing step, smoothing the individual cepstra (before extracting the cepstral peak and calculating the peak prominence), in two steps, as, first the cepstra are averaged across time, and second, the running average of cepstral magnitude is calculated across quefrency.

The CPPS was extracted through Praat software (version 6.1.41) [76], with the same parametrisation as Maryn's and Corthals' Acoustic Voice Quality Index (AVQI) Praat script (v.02.03) [78,79], i.e., for the Power Cepstrogram, pitch floor: $60 \mathrm{~Hz}$, time Step: $0.002 \mathrm{~s}$, maximum frequency: $5000 \mathrm{~Hz}$ and pre-emphasis from $50 \mathrm{~Hz}$, and the CPPS where extracted without subtracting trend before smoothing, with time averaging window: $0.01 \mathrm{~s}$, quefrency averaging window: 0.001 , peak search pitch range: $60-330 \mathrm{~Hz}$, tolerance: 0.05 , parabolic interpolation, trend line quefrency range $0-0.001 \mathrm{~s}$, straight trend type and robust fit method.

\subsection{OQ of the EGG Signals}

The contact or closed quotient (CQ) is used to compare the duration of the contact phase to the period of the vibratory cycle. It is complementary to the open quotient (OQ), since CQ and OQ ratios together constitute the total glottal cycle, and therefore CQ + OQ is always equal to 1 . DECOM is an algorithm to calculate the OQ based only on the first 
derivative of the EGG signal (DEGG). This algorithm was proposed by Henrich et al. [70] and extracts the OQ using a correlation-based method to estimate the distance between two peaks of the dEGG signal corresponding to two respective consecutive vocal folds closing instants, as well as the distance between two peaks corresponding to respective consecutive instants of vocal folds opening and closing. Howard [71] proposed a different method in which the contacting event is defined by the peak in the DEGG signal during the closing phase, while the closed phase is defined by linear quantization between samples either side of a 3:7 threshold.

For the OQ computation, a Praat script extracting OQ with both DECOM and Howard methods was used [80] and the average value for each sample with both methods was extracted.

\subsection{ABI of the Microphone Signals}

Barsties v. Latoszek's et al. multivariate acoustic model for the evaluation of breathiness [35] is more suited to dysphonic voices, as it requires both concatenated voice samples of continuous speech and a sustained vowel. In the present work, focusing mainly in evaluating intentionally breathy singing phonation, an attempt to attain deliberate continuous speech samples in various pitch regions and intensities, fully compliant with their respective GBSV samples, would probably produce disputable results. For this reason, the same sustained vowel file was given as an input for both the continuous speech and the sustained vowel sample, accepting the possibility of model inaccuracies, and therefore interpreting results with care.

For rating a pathological voice's breathiness Barsties v. Latoszek [35] proposed a combination of nine acoustic variables using stepwise multiple regression analysis: $\mathrm{ABI}=(5.0447730915-[0.172 \times \mathrm{CPPs}]-[0.193 \times \mathrm{Jit}]-[1.283 \times \mathrm{GNEmax}-4500 \mathrm{~Hz}]$ $-[0.396 \times$ Hfno $-6000 \mathrm{~Hz}]+[0.01 \times \mathrm{HNR}-\mathrm{D}]+[0.017 \times \mathrm{H} 1-\mathrm{H} 2]+[1.473 \times$ Shim $-\mathrm{dB}]-[0.088 \times$ Shim $]-[68.295 \times \mathrm{PSD}]) \times 2.9257400394$.

In the present work, the ABI was computed using the Maryn and Barsties praat script [35].

\subsection{Respiration}

Data collected from the two respiratory effort transducers (RETt and RETa) were exported as .wav files. As data recording was commenced simultaneously across data recording software, the above .wav files had identical timestamps with recorded data for the Microphone and EGG signals. All four sensor signals were loaded in four channels of a new Audacity project. Actively generated common synchronization events revealed adequate initial sensor data alignment, as well as negligible (for present study purposes) clock drift at post-trials event time. For respiratory analysis the EGG signal track was not used and was therefore temporarily disregarded. Failed trials (stopped or repeated by participant's own volition) were also disregarded and only one trial per condition was included in analysis. Valid trials were marked across sensors and labeled according to trial experimental condition. Following Salomoni et al. [22], respiratory cycles were identified visually by local maximum and minimum values of RETt and RETa data graphs to allow for optimum examination of breathing patterns.

\subsection{Phonation Duration}

Approximate phonation duration of individual trials entailing sustained both vowel and frequency was measured manually in Audacity using the selection tool and rounded to one decimal point values (sec). Glissando and arpeggio trials were excluded in order to control for in-trial pitch changes effects on phonatory duration.

\section{Results}

The above experiment data analysis revealed the results detailed below. 


\subsection{Quantitative Analysis Results}

As we can see in Table 1 the average values of the pitch difference between estimations from EGG and CM signals decrease as the breathiness rate decreases, in both frequency and mel scale results. We also observe a decrease in the average CPPS values as the breathiness rates are increasing. For the non-breathy voice samples (breathiness $=0$ ), the average CPPS value is 19.0021, while for the excessively breathy voice samples (breathiness $=4$ ), the average CPPS value is 9.7419. We can also note that the OQ values increase as the breathiness rate increase ( 0.5285 for breathiness $=0$ and 0.6473 for breathiness $=4$, using the Howard algorithm) and that the use of Howard algorithm yielded higher values compared to the DECOM method. The ABI, as expected, increases as the perceptual breathiness ratings increase.

Table 1. Descriptive outcomes: Average values for different perceptual ratings of breathiness. BR: breathiness rating, PD: Pitch Difference, MPD: Pitch Difference in Mel scale, CPPS: Smoothed Cepstral Peak Prominence, DOQ: Open Quotient with DECOM method, HOQ: Open Quotient with Howard method.

\begin{tabular}{ccccccc}
\hline BR & PD & MPD & CPPS & DOQ & HOQ & ABI \\
\hline 0 & -0.1197 & -0.1624 & 19.0021 & 0.4969 & 0.5285 & 0.1542 \\
1 & -0.5997 & -0.6998 & 16.6308 & 0.5119 & 0.5483 & 1.4276 \\
2 & -1.0749 & -1.1693 & 15.7304 & 0.5652 & 0.5778 & 2.0935 \\
3 & -2.0878 & -2.1259 & 13.2119 & 0.6067 & 0.6225 & 3.8265 \\
4 & -2.9783 & -2.9235 & 9.7419 & 0.6391 & 0.6473 & 6.1339 \\
\hline
\end{tabular}

Table 2 shows the Pearson Correlation Coefficients (r) with their $p$-values $(p)$ and Table 3 shows the Spearman Correlation Coefficients $(\rho)$ with their $p$-values $(p)$, for the PD, MPD, CPPS, and the OQ values with DECOM and Howard methods, as well as for the proposed multivariate index $\mathrm{CDH}$ and its combination with the $\mathrm{ABI}(\mathrm{CDH}+\mathrm{ABI})$. The results demonstrate higher correlation coefficients for the $\mathrm{CDH}+\mathrm{ABI}, \mathrm{CDH}$ and $\mathrm{ABI}$ indexes, while the individual parameters displaying the highest significance appear to be the HOQ and the CPPS. We observe that, with the exception of PD and MPD, all correlations (CPPS, DOQ, HOQ, $\mathrm{ABI}, \mathrm{CDH}, \mathrm{CDH}+\mathrm{ABI}$ ) were statistically significant.

Table 2. Pearson Correlation Coefficients, $r$ ( $p$-values), between the breathiness rating and the selected parameters (PD: Pitch Difference, MPD: Pitch Difference in Mel scale, CPPS: Smoothed Cepstral Peak Prominence, DOQ: Open Quotient with DECOM method, HOQ: Open Quotient with Howard method, CDH: CPPS + DOQ + HOQ, CDH + ABI: CPPS + DOQ + HOQ + ABI).

\begin{tabular}{|c|c|c|c|c|c|c|c|c|}
\hline & PD & MPD & CPPS & DOQ & HOQ & ABI & $\mathrm{CDH}$ & $\mathrm{CDH}+\mathrm{ABI}$ \\
\hline $\mathrm{r}$ & -0.0606 & -0.0494 & -0.7655 & 0.5516 & 0.6423 & 0.8107 & 0.8308 & 0.8534 \\
\hline$p$ & 0.1966 & 0.2927 & $<10^{-5}$ & $<10^{-5}$ & $<10^{-5}$ & $<10^{-5}$ & $<10^{-5}$ & $<10^{-5}$ \\
\hline
\end{tabular}

Table 3. Spearman Correlation Coefficients, $\rho$ ( $p$-Values). BR: breathiness rating, PD: Pitch Difference, MPD: Pitch Difference in Mel scale, CPPS: Cepstral Peak Prominence, DOQ: Open Quotient with DECOM method, HOQ: Open Quotient with Howard method, CDH: CPPS + DOQ + HOQ, $\mathrm{CDH}+\mathrm{ABI}: \mathrm{CPPS}+\mathrm{DOQ}+\mathrm{HOQ}+\mathrm{ABI})$.

\begin{tabular}{ccccccccc}
\hline & PD & MPD & CPPS & DOQ & HOQ & ABI & CDH & CDH + ABI \\
\hline$\rho$ & -0.1436 & -0.1427 & -0.7647 & 0.5494 & 0.6447 & 0.8279 & 0.8410 & 0.8700 \\
$p$ & 0.0021 & 0.0023 & $<10^{-5}$ & $<10^{-5}$ & $<10^{-5}$ & $<10^{-5}$ & $<10^{-5}$ & $<10^{-5}$ \\
\hline
\end{tabular}

According to the results of the Tables 1 and 2, the statistically significant individual parameters (CPPS, DOQ, and HOQ) were selected and a linear regression model was created. The resulting equation of the proposed index $(\mathrm{CDH})$, according to the coefficients of the regression, was: 
$\mathrm{CDH}=(-0.20688984 \times \mathrm{CPPS})+(-3.21878076 \times \mathrm{DOQ})+(9.56627174 \times \mathrm{HOQ})$. Adding the $\mathrm{ABI}$ values, yielded the equation:

$\mathrm{CDH}+\mathrm{ABI}=(-0.06959419 \times \mathrm{CPPS})+(-3.26429585 \times \mathrm{DOQ})+(8.27435347 \times \mathrm{HOQ})$ $+(0.2633933 \times \mathrm{ABI})$.

The regression lines between breathiness perceptual ratings and the selected parameters (CPP, $\mathrm{ABI}, \mathrm{DOQ}$, and $\mathrm{HOQ})$, and the indexes $\mathrm{CDH}$ and $\mathrm{CHD}+\mathrm{ABI}$, are shown in Figure 6.
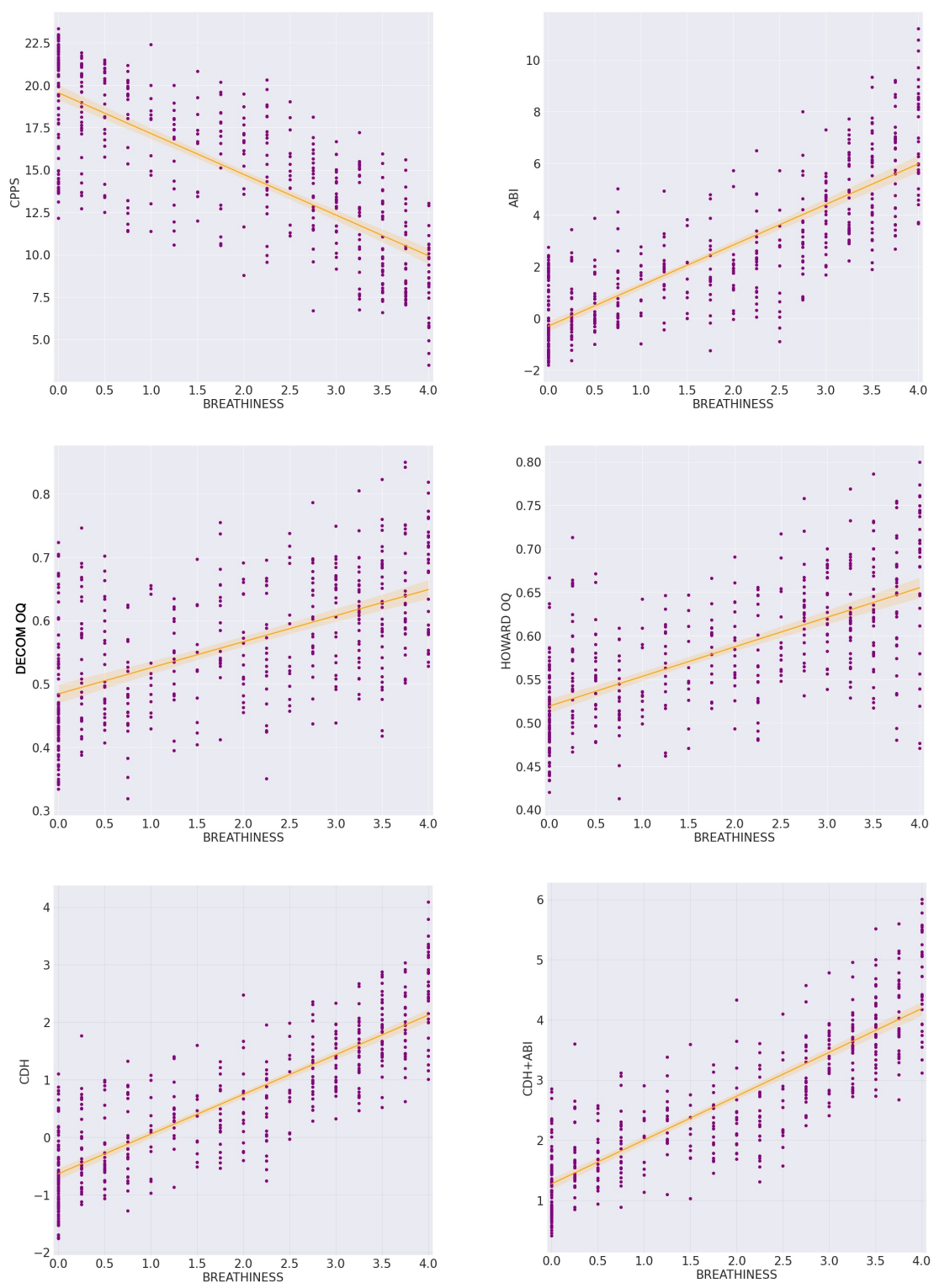

Figure 6. The scatter plots between average breathiness perceptual ratings and the selected acoustic measures: CPP $\left(\mathrm{r}=-0.7655, p<10^{-5}, \rho=-0.7647, p<10^{-5}\right)$, ABI $\left(\mathrm{r}=0.8107, p<10^{-5}, \rho=0.8279\right.$, $\left.p<10^{-5}\right)$, DOQ $\left(\mathrm{r}=0.5516, p<10^{-5}, \rho=0.5494, p<10^{-5}\right)$, HOQ $\left(\mathrm{r}=0.6423, p<10^{-5}, \rho=0.6447\right.$, $\left.p<10^{-5}\right), \mathrm{CDH}\left(\mathrm{r}=0.8308, p<10^{-5}, \rho=0.8410, p<10^{-5}\right), \mathrm{CHD}+\mathrm{ABI}\left(\mathrm{r}=0.8534, p<10^{-5}\right.$, $\rho=0.8700, p<10^{-5}$ ).

Figure 7 shows the regression lines between breathiness perceptual ratings and the difference of the pitch detection algorithm between the CM and the EGG signals (PD) with $\mathrm{r}=-0.0606, p=0.1966$, and $\rho=-0.1436, p=0.0021$, their difference converted in mel scale (MPD) with $\mathrm{r}=-0.0494, p=0.2927$, and $\rho=-0.1427, p=0.0023$, and the absolute value (ABS) of their difference (Tables 2 and 3 ). 
As we observe in Figure 7, absolute pitch difference between estimations from the CM and the EGG signals increased especially in the highest perceptual breathiness degrees. However, this pitch difference was not steadily either positive or negative. In other words, we regard that neither one of the two signals (CM and EGG) gave consistently higher or lower pitch estimates than the other.

Additionally, for the purpose of further illuminating the above relation between pitch estimation from the two signals in increasing breathiness degrees, the descriptive outcomes regarding mean absolute values of pitch difference per breathiness class in Hertz are adduced: 0.2521 (class 0), 0.7418 (class 1), 1.2193 (class 2), 2.3563 (class 3), 10.4701 (class 4). Similarly, mean values of the absolute PD as expressed in the mel scale are presented here as an indicator of the perceptual significance between the two estimation methods: 0.4020 (class 0), 1.1838 (class 1), 1.9354 (class 2), 3.6945 (class 3), 15.6859 (class 4).
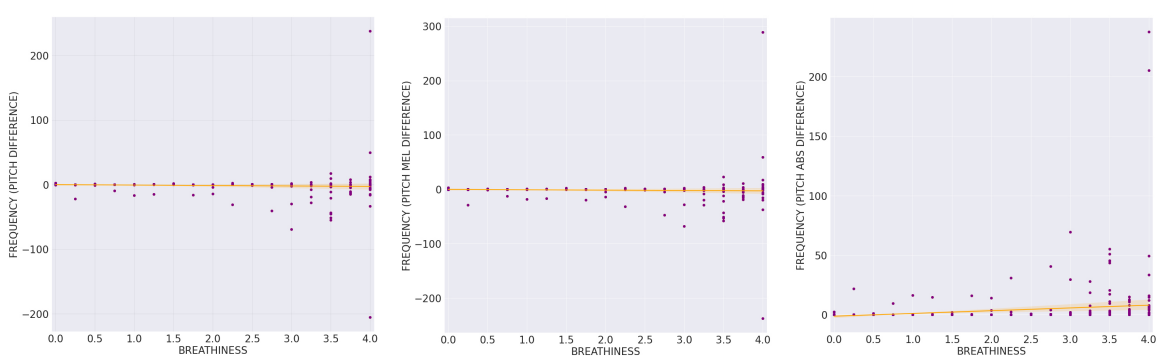

Figure 7. The scatter plots between average breathiness perceptual ratings and the difference of the pitch detection algorithm between the CM and the EGG signals (PD), with $\mathrm{r}=-0.0606, p=0.1966$, and $\rho=-0.1436, p=0.0021$, their difference converted in mel scale (MPD) with $\mathrm{r}=-0.0494, p=0.2927$, and $\rho=-0.1427, p=0.0023$, and the absolute value of their difference.

\subsection{Respiration}

Audacity wave tracks containing the trials sensor data, as described in Section 3.7, were studied and analyzed visually, in order to attain information on each participant's individual respiratory management strategies and to regard any discernible correlations to distinct trial conditions.

After studying sensor data graphs for CM, RETt, and RETa, and conducting an observatory comparison between trials, as well as between participants P1 and P2, the following patterns seem to emerge and should be taken into consideration for further investigation. For P1 an overall uniform breathing strategy was observed across trial conditions. RETt data review revealed a thoracic movement tactic that can be visually segmented into four stages (as depicted in Figure 8): (a) a thoracic region expansion, reaching a local maximum before phonation onset (stage one), (b) a slow, linear thoracic volume decrease, leading up to approximately the midpoint of trial phonation duration (stage two), (c) a consecutive significantly more rapid volume collapse (stage three), and (d) a stabilization at a local minimum briefly after stage three (stage four). 


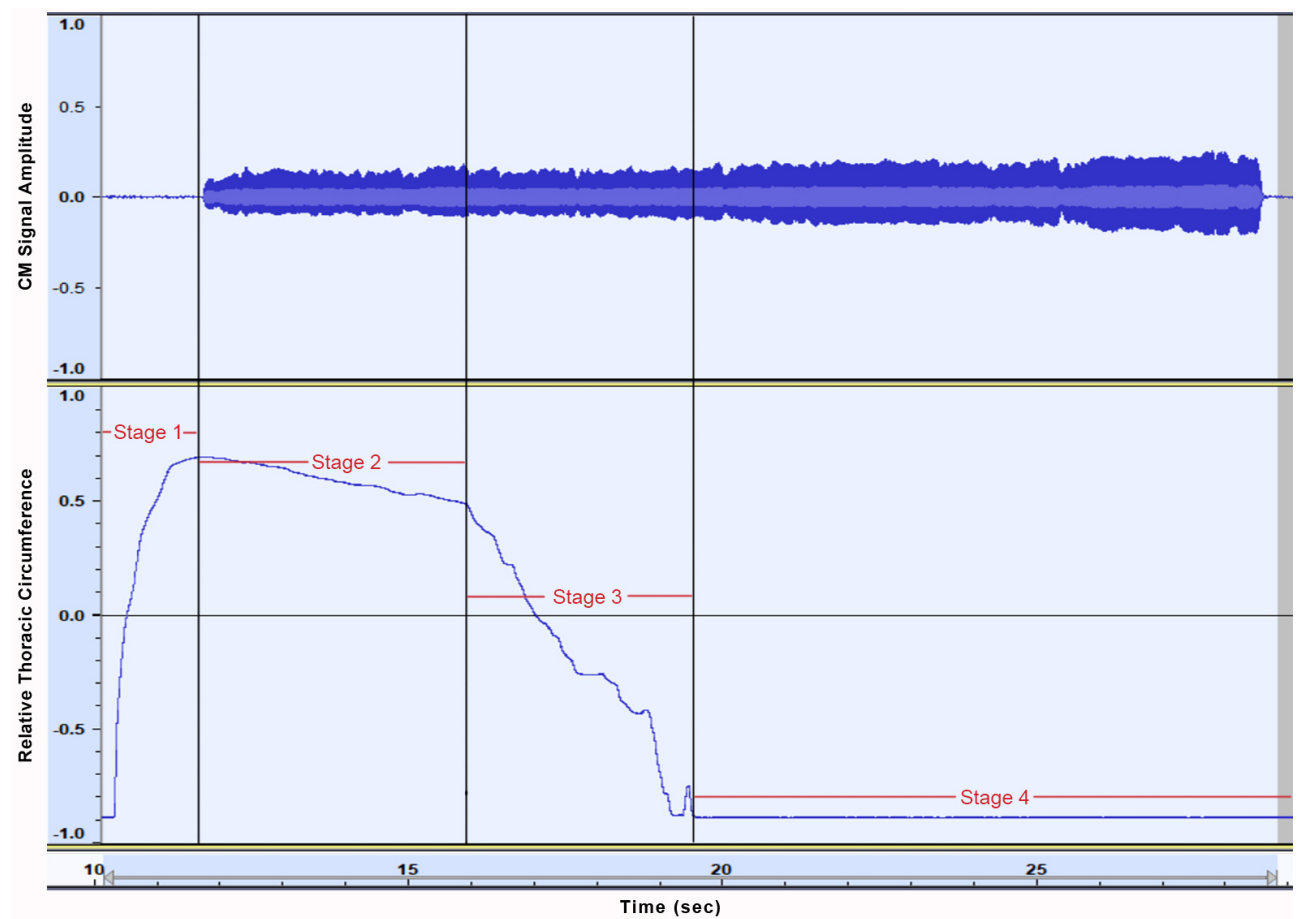

Figure 8. Graphic representation for the four distinct stages for thoracic movement during a typical phonation trial for Participant 1 (P1). Representations shown are for Condencer Microphone (CM) signal (top), and for thoracic region Respiratory Effort Transducer (RETt) (bottom).

During BSV trials, stage two of the above observations was consistently close to visual assimilation with stage three, pointing to a faster air depletion rate. This is also corroborated by mean trial durations, which were shorter in breathy phonation trials. Additionally, trials involving phonation on a high frequency seem to demonstrate a tendency for increased duration of stages two and three and thus a relatively later commencement of stage four.

RETa data for P1 showed phonation onset also on a local maximum for abdominal circumference expansion, revealing a full inspiratory capacity utilization. However, in contrast to evidence on thoracic region behavior, RETa data suggest a distinct abdominal kinetic strategy for phonation trials that varied with different breathiness degrees. More specifically, P1 showed a tendency to retain abdominal region at near maximum expansion levels for the greater part of phonation duration in trials on NBSV, and a minimal-to-no abdominal region volume decrease when approximating phonation ending. This strategy was altered in BSV trials to display an abdominal circumference attenuation shortly after phonation commencement, which continued during phonation and reached a much lower value than in NBSV trials. In GBSV trials, P1 employed a strategy ranging between the previous two, as can be seen in Figure 9.

P2 also demonstrated a personal, consistent breathing kinetic strategy, as shown in Figure 10. RETt revealed a thoracic muscle behavior generally similar to that of P1, differentiated mainly by a significantly slower thoracic volume decrease during phonation stage two. Additionally, P1 did not seem to employ a distinct approach for BSV, regarding thoracic muscle activation. RETa for P2 suggest a variation of abdominal kinetic behavior when singing trial included high frequency tones, which involved a faster inward abdominal movement with a perceptually wider displacement range. Trial breathiness degree did not seem to have an obvious effect on P2 RETa waveforms. 


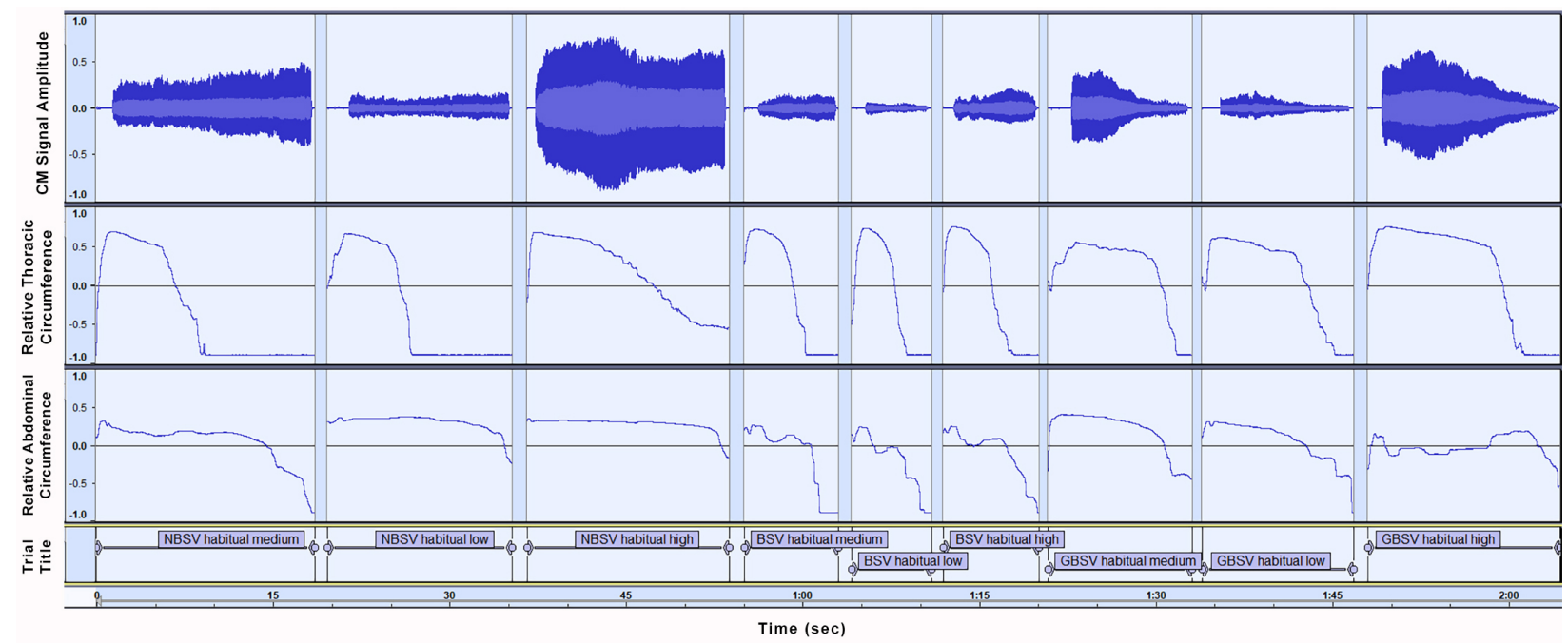

Figure 9. Graph of the nine initial trials for Participant 1 (P1). Sensors depicted are Microphone (top graph), Thoracic circumference Respiratory Effort Transducer (RETt) (middle graph), Abdominal circumference Respiratory Effort Transducer (RETta) (bottom graph). Individual trial labels correspond to trial conditions, namely (left to right) (1) breathiness degree: Non-Breathy singing Voice (NBSV), Breathy singing Voice (BSV), and 'Gradual' Breathiness singing Voice (GBSV), (2) frequency: 'habitual' refers to participant habitual voice frequency, (3) vocal intensity: medium, low, high.

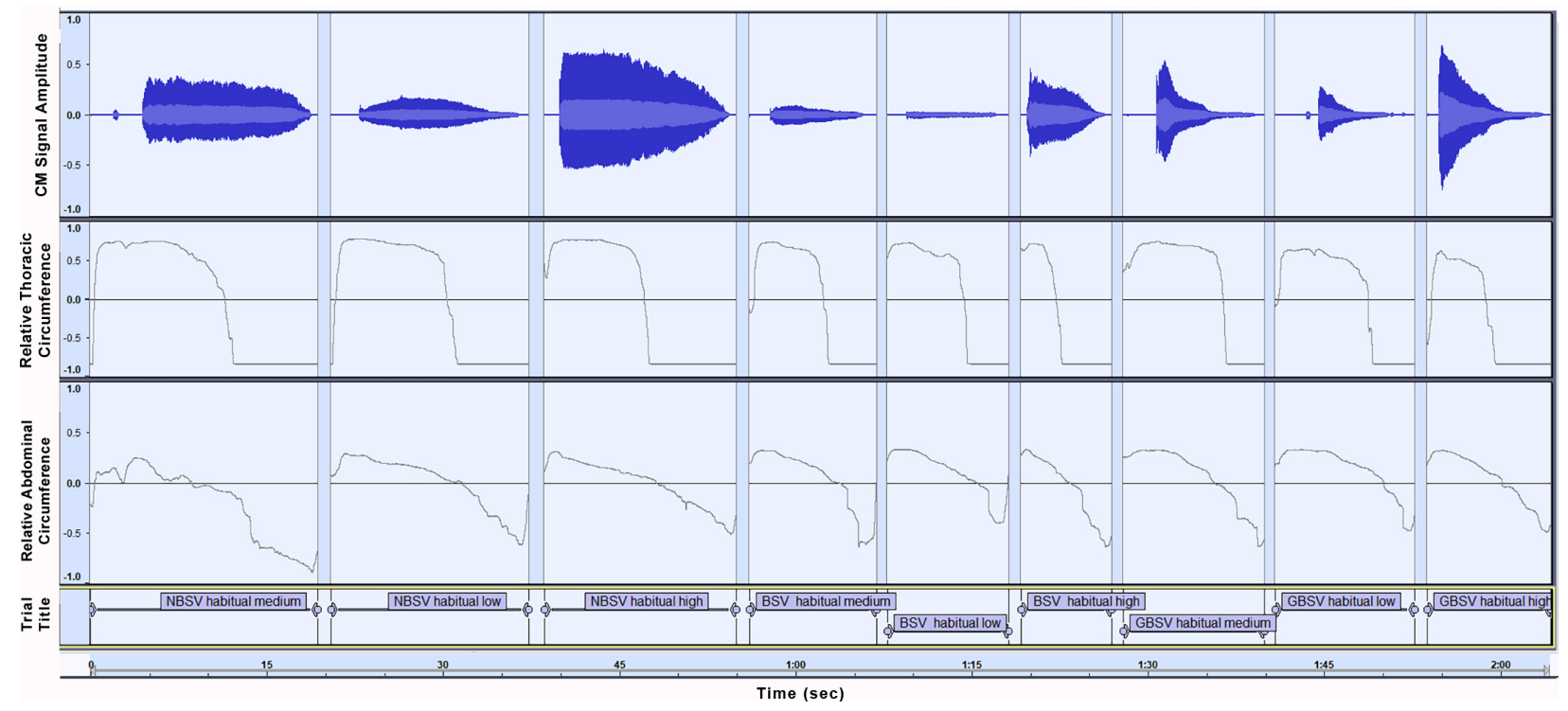

Figure 10. Graph of the nine initial trials for Participant 2 (P2). Sensors and trials illustrated are as described in corresponding Figure 9 for P1.

\subsection{Breathiness Effect on Phonation Duration}

Mean phonation duration values (in seconds) for distinct breathiness level trials were $\mathrm{NBSV}=12.43, \mathrm{GBSV}=9.43, \mathrm{BSV}=7.11$.

\section{Discussion}

The assessment study of the system-in-development described in the present work yielded the results presented within the previous sections.

\subsection{System Evaluation}

An overall evaluation of the current system revealed it to be the basis for a versatile modular tool, suitable for studio and field experimentation on the singing voice. Such a tool could also be applicable to personalized vocal education and rehabilitation. All included 
sensors are non-invasive, and experiment participants reported to have experienced no hindrance in singing and felt free to sing normally. Equipment and settings were selected such as to produce minimum noise and data synchronization was inherently sufficient. The system was able to provide data from all three major parts of the vocal mechanism that can be analyzed and correlated to further elucidate facts regarding the singing voice.

\subsection{Breathiness}

The breathiness characteristic was studied in relation with glottal and acoustic measures. Participants' glottal behavior, as monitored by the EGG sensor, confirmed the hypothesis for an increased vocal folds open quotient (or "quasi open quotient" (QOQ), as Herbst [56] suggests) in breathy voice prevalence samples. This is corroborated by high values of the ABI, low CPPS values, shorter phonation duration, and BSV trials breathing muscles gestures that seem to indicate a faster inhaled air depletion. All these factors indicate agreement with previously reported highly increased transglottal airflow in breathy vowel phonation [54]. Correlation between perceptual breathiness degree and each of the selected (acoustic, glottal, and combinatory model) parameters is illustrated in Figure 6, through depiction of the respective correlation analysis scatter plots. The combination of the ABI index together with the $\mathrm{CDH}$ index (which added EGG input data and readjusted the CPPS regression coefficient) gave the highest correlation coefficient for our dataset $\left(\mathrm{r}=0.8534, p<10^{-5}, \rho=0.8700, p<10^{-5}\right)$. Note that merely one of the variables (CPPS) used by the ABI index, when combined with two OQ extraction methods, appeared to have a strong correlation for predicting breathiness as a singing voice quality characteristic $\left(\mathrm{r}=0.8308, p<10^{-5} \rho=0.8410, p<10^{-5}\right)$. Furthermore, as expected, the ABI index showed a stronger correlation $(r=0.8107, \rho=0.8279)$ than each of the selected parameters (PD, MDP, CPPS, DOQ, HOQ) independently.

\subsection{Fundamental Frequency Estimation}

Fundamental frequency estimation deviation analysis between CM and EGG signals seems to corroborate previous studies results, comparing fundamental frequency estimation between microphone and contact sensors (accelerometres and piezoelectric contact microphone) in singing [23,81] and speech [82]. Our study confirmed "comparable results" [23] to be valid also in the comparison case between CM and EGG in normophonic (NBSV) singing voice and examined the special case of BSV, specifically their difference in frequency (PD) and mel scale (MPD). In the case of BSV, our initial hypothesis was disproved, as practically no significant deviation was detected between pitch estimation with CM and EGG signal in most degrees of vocal breathiness (the mean of PD absolute values were $<=1.2193 \mathrm{~Hz}$ for breathiness class ratings $0-2$ ).

We observe that, based on the Pearson correlation coefficient, the correlation between breathiness and PD or MPD is statistically insignificant. The significant, albeit weak, correlation emerging from the Spearman correlation coefficient could be attributed to systematical outliers, while otherwise observation of Figure 7 indicates that these variables seem to be independent for our dataset. This result could support a claim of microphone sufficiency for pitch estimation purposes, even when measuring populations of subjects with varying degrees of vocal breathiness, thus allowing its use in the ASMA vocal tuner tool. This vocal tuner is being designed for singing voice use in Elementary School Classes. Children of about this age range (6-10) have been reported to display mean fundamental frequencies of $262 \mathrm{~Hz}$ for boys and $281 \mathrm{~Hz}$ for girls, across speaking and singing tasks, with a tendency for higher frequencies during singing tasks [83]. For a voice rated with a class 2 breathiness (of the described 0-4 classification), the absolute mean difference between CM and EGG pitch estimation was found to be $1.2193 \mathrm{~Hz}$. Even in such a case this pitch deviation in a mean frequency of singing around $270 \mathrm{~Hz}$ would be the perceivable analogue of 0.077 semitones ( 7.7 Cents), while in a comfortable (for children) tone of $350 \mathrm{~Hz}$, estimation difference in a mildly breathy voice (class 1) would have a mean of $0.7418 \mathrm{~Hz}$, meaning 0.035 semitones (3.5 Cents). In his chapter "The Perception of Singing" [84], 
Sundberg describes a 2.9 cent interval at $300 \mathrm{~Hz}$ as "impossible for almost any listener to detect under any experimental conditions", and states that "the difference limen for frequency is at least 6 cents but may be considerably higher" [84]. This further supports the validity of our sensor (CM) and algorithm choice for the task at hand.

\subsection{Respiration}

Professional singers have been known to employ distinct respiratory management strategies. P1 and P2 have displayed differences in abdominal kinetic behavior during NBSV trial. However, when faced with a need for air added to the voice (BSV trials), they both opted to use an inward movement of the abdominal muscles, to apparently force an upward diaphragmatic movement and elevate subglottal air pressure. P1 and P2 RET analysis agreed with general expectation for experienced singers with years of respiratory training and indicated consistent (albeit distinct between participants) individual respiratory management strategies. However, qualitative analysis pointed to evidence of variation in extreme vocal breathiness conditions and high frequency phonation. These evidence are not generalizable, as the experiment was performed as a case study and involved intentionally breathy phonation (air added to the voice [32]) by trained professionals, and not untrained participants with inadvertently breathy or pathological voices. Nevertheless, this analysis can be used as a showcase of the proposed multi-sensor voice analysis system capability, while more cases could be the subject of further experimentation.

\subsection{Study Limitations and Future Work}

The system presented here is a work-in-progress towards a modular multi-sensor tool for singing voice analysis. A dedicated software that will record and automatically synchronize the data from all sensors (without the required use of third party or sensor manufacturer's programs) is currently in discussion. Additionally, the Kinect Azure SDK [85] is already being implemented to the system, using skeletal tracking [86] as a means of monitoring and recording (a) singers' postural alignment, and (b) kinetic habits connected to, or affecting, the production of the singing voice. This camera model has increased portability, and has been reported to have higher accuracy and lower noise than previous models, and operate in various distinct modes (e.g., different fields of view) [87]. Another possible sensor addition to this modular system is that of an accelerometer. This has been tested for the estimation of vocal measures such as fundamental frequency, glottal airflow, open quotient [27], and subglottal pressure [28].

In the present study, we have provided a brief qualitative analysis of the respiratory measurements obtained from the evaluation setup described in Sections 3.7 and 4.2. In a future work, we will provide quantitative data analyses of the outcomes from more experiments than the proof of concept results presented herein. The volume of both experimental and analytical work needed to obtain meaningful outcomes presumes a venture by far surpassing the scope of the work we have presented here. Related data have been assayed in a singer's breathing dedicated study using Principal Component Analysis (PCA) [22] and it is our intention to use similar protocols and fitting techniques for analysis, as well as appropriate low-pass filtering to eliminate high frequency instrument noise.

COVID-19-related restrictions and precaution measures led to the limitation of the current experiment's accessible and willing participant number, as the study design already required professional singers demonstrating a high expertise level in vocal control. Therefore, the experimental part of this work served mostly as a case study to assess the current system stage, and to extract predictive results regarding vocal breathiness, with professionals intentionally adding air to their voice.

Apart from the testing a stage of this in-development system, potential innovative aspects of the presented work are (i) the proposed CDH multivariate model and its combination with the ABI model, which best predicted the perceived breathiness rating for our dataset, and (ii) the comparison of pitch detection algorithm results between microphone 
and EGG signals in vocal breathiness conditions. Testing the above models and results in larger data pools has been planned.

Further extensions of the current work would also pertain to the existing dataset expansion with (i) greater participant and judges populations; (ii) participant groups of distinct vocal proficiency levels and ages; and (iii) inclusion of a number of categorized perceptual vocal features, related to voice quality, and especially vocal technique and idioms. Our goal for the integrated form of the system is for it to support multivariate correlations, using machine learning techniques and models, as well as real-time analysis and voice monitoring.

Author Contributions: Conceptualization, E.A.; methodology, E.A. and N.K.; software,N.K.; validation, A.G.; formal analysis, E.A. and N.K.; investigation, E.A. and N.K.; resources, E.A.; data curation, E.A. and N.K.; writing—original draft preparation, E.A.; writing—review and editing, N.K. and A.G.; visualization, N.K.; supervision, A.G.; project administration, E.A.; funding acquisition, A.G. All authors have read and agreed to the published version of the manuscript.

Funding: The research work was supported by the Hellenic Foundation for Research and Innovation (H.F.R.I.) under the "First Call for H.F.R.I. Research Projects to support Faculty members and Researchers and the procurement of high-cost research equipment grant" (Project Number: HFRI-FM17-3832).

Institutional Review Board Statement: The study was conducted according to the guidelines of the Declaration of Helsinki and regulations of the Ethics and Bioethics Committee of the National and Kapodistrian University of Athens.

Informed Consent Statement: Informed consent was obtained from all subjects involved in the study.

Data Availability Statement: Data available in https://github.com/nataliakotsani/Singing-VoiceMulti-Sensor-Analysis-Tool/, https:/ / doi.org/10.5281/zenodo.5732933.

Conflicts of Interest: The authors declare no conflict of interest. The funders had no role in the design of the study; in the collection, analyses, or interpretation of data; in the writing of the manuscript, or in the decision to publish the results.

\section{References}

1. Pöhlmann, E. Ancient Music in Antiquity and Beyond: Collected Essays (2009-2019); Walter de Gruyter GmbH \& Co KG: Berlin, Germany, 2020; p. 306.

2. Pestana, P.M.; Vaz-Freitas, S.; Manso, M.C. Trends in Singing Voice Research: An Innovative Approach. J. Voice 2019, 33, 263-268. [CrossRef] [PubMed]

3. Kob, M.; Henrich, N.; Herzel, H.; Howard, D.; Tokuda, I.; Wolfe, J. Analysing and Understanding the Singing Voice: Recent Progress and Open Questions. Curr. Bioinform. 2011, 6, 362-374. [CrossRef]

4. Kreiman, J.; Lee, Y.; Garellek, M.; Samlan, R.; Gerratt, B.R. Validating a psychoacoustic model of voice quality. J. Acoust. Soc. Am. 2021, 149, 457-465. [CrossRef] [PubMed]

5. Samlan, R.A.; Story, B.H.; Bunton, K. Relation of perceived breathiness to laryngeal kinematics and acoustic measures based on computational modeling. J. Speech Lang. Hear. Res. 2014, 56, 1209-1223. [CrossRef]

6. Sundberg, J. The Science of the Singing Voice; Northern Illinois University Press: DeKalb, IL, USA, 1987; p. 216.

7. Titze, I.R. Nonlinear source-filter coupling in phonation: Theory. J. Acoust. Soc. Am. 2008, 123, 2733-2749. [CrossRef]

8. Kob, M. Singing voice modeling as we know it today. Acta Acust. United Acust. 2004, 90, 649-661.

9. Muda, L.; Begam, M.; Elamvazuthi, I. Voice Recognition Algorithms using Mel Frequency Cepstral Coefficient (MFCC) and Dynamic Time Warping (DTW) Techniques. J. Comput. 2010, 2, 138-143.

10. Angelakis, E.; Andreopoulou, A.; Georgaki, A. Multisensory biofeedback: Promoting the recessive somatosensory control in operatic singing pedagogy. Biomed. Signal Process. Control. 2021, 66, 102400. [CrossRef]

11. Boersma, P.; Weenink, D. Praat: Doing Phonetics by Computer [Computer Program]; University of Amsterdam: Amsterdam, The Netherlands, 2021.

12. Andreopoulou, A.; Kotsani, N.; Dedousis, G.; Georgaki, A. Evaluating the vocal characteristics of elementary school students: Basic assessment tools and methodology. In Proceedings of the Interaction Design and Children, IDC 2021, Athens, Greece, 24-30 June 2021; pp. 216-223. [CrossRef]

13. Jang, S.J.; Choi, S.H.; Kim, H.M.; Choi, H.S.; Yoon, Y.R. Evaluation of Performance of Several Established Pitch Detection Algorithms in Pathological Voices. In Proceedings of the 29th Annual International Conference of the IEEE EMBS, Lyon, France, 22-26 August 2007; pp. 620-623. 
14. Lopes, L.W.; Barbosa Lima, I.L.; Alves Almeida, L.N.; Cavalcante, D.P.; De Almeida, A.A.F. Severity of voice disorders in children: Correlations between perceptual and acoustic data. J. Voice 2012, 26, 819.e7-819.e12, [CrossRef]

15. Oliveira, R.C.; Teixeira, L.C.; Gama, A.C.C.; de Medeiros, A.M. Auditory-perceptive, acoustic and vocal self-perception analyses in children. J. Soc. Bras. Fonoaudiol. 2011, 23, 158-163. [CrossRef]

16. Eddins, D.A.; Anand, S.; Camacho, A.; Shrivastav, R. Modeling of Breathy Voice Quality Using Pitch-strength Estimates. J. Voice 2016, 30, 774.e1-774.e7, [CrossRef]

17. Glottal. Available online: http:/ /www.glottal.com/PhaseComp.html (accessed on 28 August 2021).

18. VoceVista. VoceVista: Software and Hardware for Singers; VoceVista: Eugene, OR, USA, 2009.

19. Shue, Y.L.; Keating, P.; Vicenik, C.; Kristine, Y. VoiceSause: A Program for Voice Analysis. In Proceedings of the 17th International Congress of Phonetic Sciences, Hong Kong, China, 17-21 August 2011; Volume 3, pp. 1846-1849.

20. Tehrani, H. EGGWorks: A Program for Automated Analysis of EGG Signals. 2009. Available online: https://appsobabble.com/ functions/EGGWorks.aspx (accessed on 28 August 2021).

21. Watson, P.J.; Hixon, T.J. Respiratory kinematics in classical (opera) singers. J. Speech Hear. Res. 1985, 28, 104-122.10.1044/jshr.2801.104. [CrossRef] [PubMed]

22. Salomoni, S.; Van Den Hoorn, W.; Hodges, P. Breathing and singing: Objective characterization of breathing patterns in classical singers. PLoS ONE 2016, 11, e0155084, [CrossRef]

23. Castellana, A.; Leocata, I.; Carullo, A.; Astolfi, A. Singing voice quality assessment in professional singers using microphones and contact sensors. In Proceedings of the Euronoise 2018-Conference Proceedings, Heraklion, Greece, 27-31 May 2018; pp. 1849-1854.

24. European Commission. Saving our Cultural Heritage; European Commission: Brussels, Belgium, 2020.

25. Chawah, P.; Al Kork, S.K.; Fux, T.; Adda-Decker, M.; Amelot, A.; Audibert, N.; Denby, B.; Dreyfus, G.; Jaumard-Hakoun, A.; Pillot-Loiseau, C.; et al. An educational platform to capture, visualize and analyze rare singing. In Proceedings of the Annual Conference of the International Speech Communication Association, Singapore, 14-18 September 2014; pp. $2128-2129$.

26. Griffin, B.; Woo, P.; Colton, R.; Casper, J.; Brewer, D. Physiological characteristics of the supported singing voice. A preliminary study. J. Voice 1995, 9, 45-56. [CrossRef]

27. Cortés, J.P.; Espinoza, V.M.; Ghassemi, M.; Mehta, D.D.; Van Stan, J.H.; Hillman, R.E.; Guttag, J.V.; Zañartu, M. Ambulatory assessment of phonotraumatic vocal hyperfunction using glottal airflow measures estimated from neck-surface acceleration. PLoS ONE 2018, 13, e209017. [CrossRef]

28. Marks, K.L.; Lin, J.Z.; Fox, A.B.; Toles, L.E.; Mehta, D.D. Impact of Nonmodal Phonation on Estimates of Subglottal Pressure From Neck-Surface Acceleration in Healthy Speakers. J. Speech Lang. Hear. Res. 2019, 62, 3339-3358. [CrossRef] [PubMed]

29. Toles, L.E.; Ortiz, A.J.; Marks, K.L.; Mehta, D.D.; Van Stan, J.H.; Hillman, R.E. Amount and Characteristics of Speaking and Singing Voice Use in Vocally Healthy Female College Student Singers During a Typical Week. J. Voice 2020. [CrossRef]

30. Toles, L.E.; Ortiz, A.J.; Marks, K.L.; Burns, J.A.; Hron, T.; Stan, J.H.V.; Mehta, D.D.; Hillman, R.E. Differences Between Female Singers With Phonotrauma and Vocally Healthy Matched Controls in Singing and Speaking Voice Use During 1 Week of Ambulatory Monitoring. Am. J. Speech Lang. Pathol. 2021, 30, 199-209. [CrossRef] [PubMed]

31. Ortiz, A.J.; Toles, L.E.; Marks, K.L.; Capobianco, S.; Mehta, D.D.; Hillman, R.E.; Van Stan, J.H. Automatic speech and singing classification in ambulatory recordings for normal and disordered voices. J. Acoust. Soc. Am. 2019, 146, EL22-EL27. [CrossRef] [PubMed]

32. Aaen, M.; McGlashan, J.; Thu, K.T.; Sadolin, C. Assessing and Quantifying Air Added to the Voice by Means of Laryngostroboscopic Imaging, EGG, and Acoustics in Vocally Trained Subjects. J. Voice 2021, 35, 326.e1-326.e11, [CrossRef] [PubMed]

33. Ternström, S.; D'Amario, S.; Selamtzis, A. Effects of the Lung Volume on the Electroglottographic Waveform in Trained Female Singers. J. Voice 2020, 34, 485-e1. [CrossRef]

34. Scherer, R.C.; Titze, I.R. The Abduction Quotient Related to Vocal Quality. J. Voice 1987, 1, 246-251. [CrossRef]

35. Latoszek, B.B.V.; Maryn, Y.; Gerrits, E.; De Bodt, M. The Acoustic Breathiness Index (ABI): A Multivariate Acoustic Model for Breathiness. J. Voice 2017, 31, 511.e11-511.e27. [CrossRef]

36. Childers, D.G.; Lee, C.K. Vocal quality factors: Analysis, synthesis, and perception. J. Acoust. Soc. Am. 1991, 90, 2394-2410. [CrossRef] [PubMed]

37. Kempster, G.B.; Gerratt, B.R.; Abbott, K.V.; Barkmeier-Kraemer, J.; Hillman, R.E. Consensus auditory-perceptual evaluation of voice: Development of a standardized clinical protocol. Am. J. Speech Lang. Pathol. 2009, 18, 124-132. [CrossRef]

38. Hillenbrand, J.; Houde, R.A. Acoustic correlates of breathy vocal quality: Dysphonic voices and continuous speech. J. Speech Lang. Hear. Res. 1996, 39, 311-321. [CrossRef] [PubMed]

39. Fritzell, B.; Hammarberg, B.; Gauffin, J.; Karlsson, I.; Sundberg, J. Breathiness and insufficient vocal fold closure. J. Phon. 1986, 14, 549-553. [CrossRef]

40. Latoszek, B.B.V.; Kim, G.H.; Delgado Hernández, J.; Hosokawa, K.; Englert, M.; Neumann, K.; Hetjens, S. The validity of the Acoustic Breathiness Index in the evaluation of breathy voice quality: A Meta-Analysis. Clin. Otolaryngol. 2021, 46, 31-40. [CrossRef] [PubMed]

41. Yu, M. Vocal Exercises for Reducing Vocal Damages Caused. J. Korean Soc. Laryngol. Phoniatr. Logop. 2017, 28, 14-16. [CrossRef]

42. Edgerton, M.E. The 21st-Century Voice: Contemporary and Traditional Extra-Normal Voice, 2nd ed.; Scarecrow Press: Lanham, MD, USA, 2015; p. 230. 
43. Graham, E.; Angadi, V.; Sloggy, J.; Stemple, J. Contribution of glottic insufficiency to perceived Breathiness in classically trained singers. Med. Probl. Perform. Artist. 2016, 31, 179-184. [CrossRef]

44. Shrivastav, R.; Eddins, D.A.; Anand, S. Pitch strength of normal and dysphonic voices. J. Acoust. Soc. Am. 2012, 131, 2261-2269. [CrossRef]

45. Pongweni, A.J. An acoustic study of the qualitative and pitch effect of breathy-voice on Shona vowels. J. Phon. 1983, 11, 129-138 [CrossRef]

46. Hartl, D.M.; Hans, S.; Vaissière, J.; Brasnu, D.F. Objective acoustic and aerodynamic measures of breathiness in paralytic dysphonia. Eur. Arch. Oto-Rhino-Laryngol. 2003, 260, 175-182. [CrossRef]

47. Shrivastav, R.; Sapienza, C.M. Objective measures of breathy voice quality obtained using an auditory model. J. Acoust. Soc. Am. 2003, 114, 2217-2224. [CrossRef] [PubMed]

48. Teixeira, J.P.; Oliveira, C.; Lopes, C. Vocal Acoustic Analysis-Jitter, Shimmer and HNR Parameters. Procedia Technol. 2013, 9, 1112-1122. [CrossRef]

49. Fraile, R.; Godino-Llorente, J.I. Cepstral peak prominence: A comprehensive analysis. Biomed. Signal Process. Control. 2014, 14, 42-54. [CrossRef]

50. Borsky, M.; Mehta, D.D.; Gudjohnsen, J.P.; Gudnason, J. Classification of voice modality using electroglottogramwave forms. In Proceedings of the Annual Conference of the International Speech Communication Association, San Francisco, CA, USA, 8-12 September 2016. [CrossRef]

51. Kadiri, S.R.; Yegnanarayana, B. Breathy to tense voice discrimination using zero-time windowing cepstral coefficients (ZTWCCs). In Proceedings of the Annual Conference of the International Speech Communication Association, Hyderabad, India, 2-6 September 2018; pp. 232-236. [CrossRef]

52. Tylečková, L.; Skarnitzl, R. The mapping of voice parameters in connected speech of healthy Common Czech male speakers. Akust. Listy 2019, 25, 10-18.

53. Murton, O.; Hillman, R.; Mehta, D. Cepstral peak prominence values for clinical voice evaluation. Am. J. Speech-Lang. Pathol. 2020, 29, 1596-1607. [CrossRef] [PubMed]

54. Klatl, D.H.; Klati, L.C. Analysis, synthesis, and perception of voice quality variations among female and male talkers. J. Acoust. Soc. Am. 1990, 87, 820-857. [CrossRef]

55. BIOPAC. Product Sheet—SS5LB Respiratory Effort Transducer; BIOPAC Systems Inc.: Goleta, CA, USA, 2018.

56. Herbst, C.T. Electroglottography-An Update. J. Voice 2020, 34, 503-526. [CrossRef]

57. Rothenberg, M. A multichannel electroglottograph. J. Voice 1992, 6, 36-43. [CrossRef]

58. Herbst, C.; Ternström, S. A comparison of different methods to measure the EGG contact quotient. Logop. Phoniatr. Vocol. 2006, 31, 126-138. [CrossRef]

59. Titze, I.R. Interpretation of the electroglottographic signal. J. Voice 1990, 4, 1-9. [CrossRef]

60. Michaud, A. Mode d'emploi de l'électroglottographe EG2-PC du Laboratoire de Phonétique et Phonologie. 2005. Available online: http:/ / voiceresearch.free.fr/egg/mode-emploi-EGG-LPP.pdf (accessed on 28 November 2021).

61. Deliyski, D.D.; Shaw, H.S.; Evans, M.K. Adverse effects of environmental noise on acoustic voice quality measurements. J. Voice 2005, 19, 15-28. [CrossRef]

62. Deliyski, D.D.; Shaw, H.S.; Evans, M.K.; Vesselinov, R. Regression tree approach to studying factors influencing acoustic voice analysis. Folia Phoniatr. Logop. 2006, 58, 274-288. [CrossRef] [PubMed]

63. Massaroni, C.; Nicolò, A.; Presti, D.L.; Sacchetti, M.; Silvestri, S.; Schena, E. Contact-based methods for measuring respiratory rate. Sensors 2019, 19, 908. [CrossRef] [PubMed]

64. Wlodarczak, M. RespInPeace: Toolkit for Processing Respiratory Belt Data. In Proceedings of the FONETIK 2019 Stockholm, Stockholm, Sweden, 10-12 June 2019; pp. 115-118.

65. Zarate, J.M. The neural control of singing. Front. Hum. Neurosci. 2013, 7, 237. [CrossRef]

66. Kleber, B.; Friberg, A.; Zeitouni, A.; Zatorre, R. Experience-dependent modulation of right anterior insula and sensorimotor regions as a function of noise-masked auditory feedback in singers and nonsingers. NeuroImage 2017, 147, 97-110. [CrossRef]

67. Shaabana, A.L.A.; Zheng, R. CRONOS: A Post-hoc Data Driven Multi-Sensor. ACM Trans. Sens. Netw. (TOSN) 2019, 15, 1-20. [CrossRef]

68. Stoller, D.; Dixon, S. Analysis and classification of phonation modes in singing. In Proceedings of the 17th International Society for Music Information Retrieval Conference, ISMIR 2016, New York, NY, USA, 7-11 August 2016; pp. 80-86.

69. Kotsani, N. Singing-Voice-Multi-Sensor-Analysis-Tool (Version v1.0). 2021. Available online: https://zenodo.org/record/573293 3\#.YaN7PSORppQ (accessed on 28 November 2021).

70. Henrich, N.; d'Alessandro, C.; Doval, B.; Castellengo, M. On the use of the derivative of electroglottographic signals for characterization of nonpathological phonation. J. Acoust. Soc. Am. 2004, 115, 1321-1332. [CrossRef]

71. Howard, D.M. Variation of Electrolaryngographically Derived Closed Quotient for Trained and Untrained Adult Female Singers. J. Voice 1995, 9, 163-172. [CrossRef]

72. Sofranko, J.L.; Prosek, R.A. The effect of experience on classification of voice quality. J. Voice 2012, 26, 299-303.10.1016/j.jvoice.2011.07.003. [CrossRef]

73. Lee, M.; Drinnan, M.; Carding, P. The reliability and validity of patient self-rating of their own voice quality. Clin. Otolaryngol. 2005, 30, 357-361. [CrossRef] [PubMed] 
74. Vieira, M.N.; McInnes, F.R.; Jack, M.A. On the influence of laryngeal pathologies on acoustic and electroglottographic jitter measures. J. Acoust. Soc. Am. 2002, 111, 1045-1055. [CrossRef] [PubMed]

75. Jadoul, Y.; Thompson, B.; de Boer, B. Introducing Parselmouth: A Python interface to Praat. J. Phon. 2018, 71, 1-15. [CrossRef]

76. Boersma, P.; Van Heuven, V. Speak and unSpeak with PRAAT RAAT. Available online: https://www.fon.hum.uva.nl/paul/ papers/speakUnspeakPraat_glot2001.pdf (accessed on 28 November 2021).

77. Watts, C.R.; Awan, S.N.; Maryn, Y. A Comparison of Cepstral Peak Prominence Measures From Two Acoustic Analysis Programs. J. Voice 2017, 31, 1-387. [CrossRef]

78. Maryn, Y.; De Bodt, M.; Barsties, B.; Roy, N. The value of the Acoustic Voice Quality Index as a measure of dysphonia severity in subjects speaking different languages. Eur. Arch. Oto-Rhino-Laryngol. 2014, 271, 1609-1619. [CrossRef]

79. Maryn, Y. Praat-Script-Avqi-v0203. Available online: https://www.vvl.be/documenten-en-paginas/praat-script-avqi-v0203 (accessed on 28 November 2021).

80. GitHub. Kirbyj/Praatdet: Praat-Based Tools for EGG Analysis; GitHub: San Francisco, CA, USA, 2020.

81. Coleman, R.F. Comparison of microphone and neck-mounted accelerometer monitoring of the performing voice. J. Voice 1988, 2, 200-205. [CrossRef]

82. Mehta, D.D.; Van Stan, J.H.; Hillman, R.E. Relationships between vocal function measures derived from an acoustic microphone and a subglottal neck-surface accelerometer. IEEE/ACM Trans. Audio Speech Lang. Process. 2016, 24, 659-668. [CrossRef]

83. Sorenson, D.N. A fundamental frequency investigation of children ages 6-10 years old. J. Commun. Disord. 1989, 22, 115-123. [CrossRef]

84. Sundberg, J. The Perception of Singing. In The Psychology of Music, 2nd ed.; Deutch, D., Ed.; Academic Press: Cambridge, MA, USA, 1999; Chapter 6, pp. 171-214. [CrossRef]

85. Azure Kinect DK-Develop AI Models I Microsoft Azure. Available online: https://azure.microsoft.com/en-us/services/kinectdk/\#overview (accessed on 28 August 2021).

86. Albert, J.A.; Owolabi, V.; Gebel, A.; Brahms, C.M.; Granacher, U.; Arnrich, B. Evaluation of the pose tracking performance of the azure kinect and kinect v2 for gait analysis in comparison with a gold standard: A pilot study. Sensors 2020, 20, 5104. [CrossRef] [PubMed]

87. Tölgyessy, M.; Dekan, M.; Chovanec, L. Skeleton tracking accuracy and precision evaluation of Kinect V1, Kinect V2, and the azure kinect. Sensors 2021, 21, 413. [CrossRef] [PubMed] 